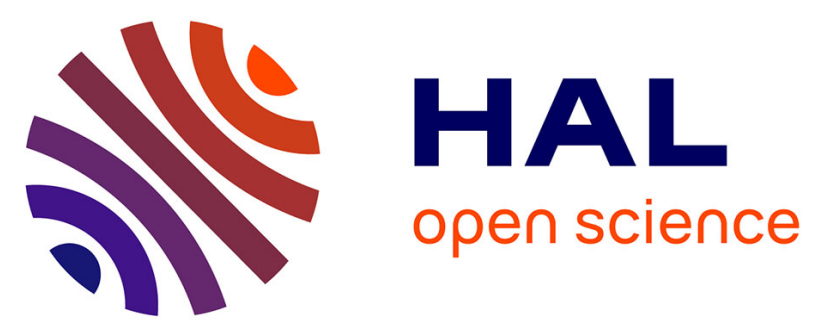

\title{
MMS observations of intense whistler waves within Earth's supercritical bow shock: Source mechanism and impact on shock structure and plasma transport
}

\author{
A. Hull, Laurent Muschietti, O. Le Contel, J. Dorelli, P.-E. Lindqvist
}

\section{- To cite this version:}

A. Hull, Laurent Muschietti, O. Le Contel, J. Dorelli, P.-E. Lindqvist. MMS observations of intense whistler waves within Earth's supercritical bow shock: Source mechanism and impact on shock structure and plasma transport. Journal of Geophysical Research Space Physics, 2020, 125 (7), pp.e2019JA027290. 10.1029/2019JA027290 . insu-02571333

\section{HAL Id: insu-02571333}

https://hal-insu.archives-ouvertes.fr/insu-02571333

Submitted on 29 Mar 2021

HAL is a multi-disciplinary open access archive for the deposit and dissemination of scientific research documents, whether they are published or not. The documents may come from teaching and research institutions in France or abroad, or from public or private research centers.
L'archive ouverte pluridisciplinaire HAL, est destinée au dépôt et à la diffusion de documents scientifiques de niveau recherche, publiés ou non, émanant des établissements d'enseignement et de recherche français ou étrangers, des laboratoires publics ou privés. 


\section{JGR Space Physics}

\author{
RESEARCH ARTICLE \\ 10.1029/2019JA027290 \\ Key Points: \\ - Whistlers near lower-hybrid \\ frequencies are consistent with the \\ modified two-stream instability \\ due to the reflected ion and electron \\ drift \\ - Whistlers are associated with large \\ currents that can excite electrostatic \\ waves and contribute to energy \\ exchange processes within shocks \\ - Lower-hybrid whistler waves \\ interact with the electrons and \\ induce anisotropies that can drive \\ quasiparallel high-frequency \\ whistlers
}

Correspondence to:

A. J. Hull,

ahull@ssl.berkeley.edu

Citation:

Hull, A. J., Muschietti, L., Le Contel, O., Dorelli, J. C., \& Lindqvist, P.-A. (2020). MMS observations of intense whistler waves within Earth's supercritical bow shock: Source mechanism and impact on shock structure and plasma transport. Journal of Geophysical Research: Space Physics, 125, e2019JA027290. https://doi.org/10. 1029/2019JA027290

Received 12 AUG 2019 Accepted 21 APR 2020 Accepted article online 6 MAY 2020

(C)2020. American Geophysical Union. All Rights Reserved.

\section{MMS Observations of Intense Whistler Waves Within Earth's Supercritical Bow Shock: Source Mechanism and Impact on Shock Structure and Plasma Transport}

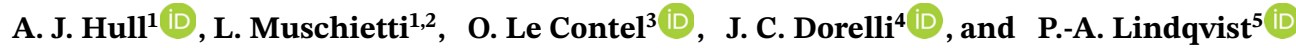 \\ ${ }^{1}$ Space Sciences Laboratory, University of California Berkeley, Berkeley, Ca, USA, ${ }^{2}$ LATMOS-IPSL-UVSQ-CNRS, \\ Guyancourt, France, ${ }^{3}$ Laboratoire de Physique des Plasmas, UMR7648, CNRS, Ecole Polytechnique, UPMC, Université \\ Paris 06, University of Paris-Sud, Paris, France,${ }^{4}$ NASA Goddard Space Flight Center, Greenbelt, MD, USA, \\ ${ }^{5} \mathrm{KTH}$, Royal Institute of Technology, Stockholm, Sweden
}

Abstract The properties of whistler waves near lower-hybrid frequencies within Earth's quasi-perpendicular bow shock are examined using data from the Magnetospheric Multiscale (MMS) mission. These waves appear as right-hand polarized wave packets propagating upstream obliquely to the magnetic field and shock normal with phase speeds from a few hundred up to $1,600 \mathrm{~km} / \mathrm{s}$. The wavelengths are near the ion inertial length scale $\left(\lambda \sim 0.3-1.3 \lambda_{i}\right)$. Detailed analysis finds characteristics consistent with the modified two-stream instability mechanism driven by the reflected ion and electron drift. Correlations between wave and electron anisotropy variations reveal that the whistlers are affecting electron dynamics and thus their perpendicular and parallel temperatures. The electron signatures are explainable via the interaction of magnetized electrons in the whistler induced nonmonotonic magnetic fields. These waves have intense magnetic fields $\left(\delta B / B_{\circ} \sim 0.1-1\right)$ and carry sizable currents that are a significant fraction of the thermal current $\left(\left|J / J_{\text {vte }}\right| \sim 0.1-0.5\right)$. The whistler-induced currents and the electron anisotropies are sufficiently large to respectively excite high-frequency (HF) electrostatic $(>100 \mathrm{~Hz})$ and $\mathrm{HF}$ whistler waves $\left(f \sim 0.1-0.5 f_{\text {ce }}\right)$. Energy dissipation $\mathbf{J} \cdot \mathbf{E}$ from whistlers at $30 \mathrm{~Hz}$ and below range from a few thousandths to few hundredths of $\mu \mathrm{W} / \mathrm{m}^{3}$. Comparisons reveal that plasma energy is converted to wave energy in the foot, whereas wave energy gets dissipated into the plasma in the ramp, where irreversible heating occurs. These observed features are indicative of an intricate coupling between small-scale interaction processes and larger-scale structure transpiring within the layer. Such a characterization is only made possible now with the MMS high-time-resolution measurements.

\section{Introduction}

Collisionless fast mode shocks play an important role in converting kinetic energy of the plasma flow into thermal energy. This conversion process occurs within a layer that is much shorter than the collisional mean free path, hence the "collisionless" denomination. They are also important sites for the acceleration of plasma particles to high energies and the generation of electromagnetic and electrostatic waves, which can act to energize and thermalize the plasma. Whistler waves are well-known to be an important part of the shock macroscopic structure, and thus have garnered a considerable amount of attention in theoretical (e.g., Krasnoselskikh et al., 2002; Tidman \& Krall, 1971) and observational studies within or upstream of Earth's bow shock (e.g., Bale et al., 2005; Balikhin, de Wit et al., 1997; Dimmock et al., 2013; Fairfield, 1974; Hull et al., 2012; Krasnosel'skikh et al., 1991; Krasnoselskikh et al., 2013; Lembege et al., 2004; Oka et al., 2017; Rodriguez \& Gurnett, 1975; Walker et al., 1999; Wilson, 2016; Zhang et al., 1999, and references therein), interplanetary shocks (e.g., Lengyel-Frey et al., 1996; Wilson et al., 2013, 2017), and also at planetary bow shocks, such as at Mercury (e.g., Fairfield \& Behannon, 1976), Uranus (e.g., Smith et al., 1991), and Venus (e.g., Orlowski \& Russell, 1991). Despite much attention, the generation mechanisms, their role in shock internal structure, and consequences to plasma transport are not fully understood. This is particularly the case within high-Mach number supercritical shock layers, where the whistlers at lower-hybrid frequencies and below are more intense and often found to be obliquely propagating with respect to the background magnetic field $\mathbf{B}_{\circ}$. Owing to their large amplitude magnetic fields $\left(\delta B / B_{\circ} \sim 0.1-1\right.$.), such intense 
whistlers can significantly modify the shock layer, adding nonmonotonic features to the ramp that can affect particle transport. For example, their interaction with electrons may induce anisotropies, which can induce secondary high-frequency whistler waves (Hull et al., 2012). The large magnetic fields of the whistlers also imply sizable, subion inertial length scale currents, which to date have not been fully studied. Such currents in asymmetric and/or appropriately phased electric fields can lead to "anomalous" dissipation ( $<\mathbf{J} \cdot \mathbf{E}>>0)$ (Wilson et al., 2014a, 2014b). If they are sufficiently large, the currents can also provide a free energy source for waves, such as large amplitude electrostatic waves, which can further modify the shock structure and influence plasma transport. In the past the gross currents within shock layers were found to be insufficient to drive various current sourced instabilities in the ramp (e.g., Scudder, Mangeney, Lacombe, Harvey, Aggson, et al.,1986). However, recent studies are indicating the contrary due to the currents being concentrated on smaller spatial scales than previously thought (e.g., Wilson et al., 2014a, 2014b).

Source mechanisms for the intense whistlers are under current debate, particularly near lower-hybrid frequencies. One possibility is that the whistler waves may be intrinsically generated by nonlinearities in a steepening shock front. These are emitted upstream away from the shock preferentially along the shock normal (Krasnoselskikh et al., 2002). In this picture dispersive effects are dominant and viewed to play an essential role in the formation and stability of the shock front. At low enough Mach numbers, it is recognized that dispersive emission is enough to balance the nonlinear growth of the shock, enabling it to be stationary. However, if the Mach number gets too high, this model predicts that dispersive radiation is unable to balance the shock nonlinear growth in a stationary way. The shock undergoes a process of cyclic reformation, whereby periodic bursts of nonlinear whistler waves are emitted upstream. Observations of whistlers upstream and within the shock ramp interpreted to be consistent with dispersive radiation have been reported (e.g., Balikhin, de Wit et al., 1997; Balikhin, Walker, et al., 1997; Dimmock et al., 2019; Lobzin et al., 2007; Sundkvist et al., 2012; Walker et al., 1999). Signatures collectively reported to be consistent with dispersive induced emission or reformation process upstream and/or within the shock include the presence or time-development of whistler substructure or whistler bursts, upstream directed wave vectors preferentially oriented toward the normal in the plasma frame, wavelengths that can reach down to the electron inertial scale, and upstream directed Poynting fluxes.

Alternatively, intense whistlers in the lower-hybrid regime may be generated by microinstabilities driven by the relative drift between electrons and ions. Included in this category are the modified two- stream instabilities (MTSI) and lower-hybrid drift instability (e.g., Scudder, Mangeney, Lacombe, Harvey, Wu, et al., 1986; Wu et al., 1984). Of these, the MTSI (also referred to as the kinetic cross-field streaming instability Wu et al., 1983) appears to be a favored candidate in the foot and into the first part of the shock ramp and has received much attention in theoretical and simulation studies (Hellinger \& Mangeney, 1997; Matsukiyo \& Scholer, 2003, 2006; Muschietti \& Lembège, 2017). It is important to emphasize that MTSI is not referring to the usual electrostatic limit corresponding to propagation angles $\theta_{\mathrm{kb}} \sim 90^{\circ}$ with respect to the background magnetic field $B_{\circ}$ but refers to the more generalized description for oblique propagation whereby electromagnetic effects become important, if not dominant. It is well-known that ion reflection occurs at supercritical shocks, which gives rise to ion distributions composed of an incoming solar wind component and a reflected beam. Thus, there are two distinct free energy sources at play for the MTSI. One source is the relative drift between the incoming solar wind ions and the electrons, which is relatively slow (on the order of the Alfvén speed $V_{A}$ ), and points toward the ramp. The second free energy source is the relative drift between the reflected ion beam and the electrons, which is much larger than $V_{A}$ and has an upstream directed component. Correspondingly, two types of whistlers with different characteristics can be excited. The first type of whistlers, which are associated with the slow drift, are quasi-perpendicular and have wavelengths several times the electron inertial length. These propagate toward the ramp at an angle a few degrees off of $90^{\circ}$ with respect to $\mathbf{B}_{\text {. }}$. The second type of whistlers, which are associated with the fast drift, have wavelengths that are near the ion inertial length $\lambda_{i}=c / \omega_{p i}$. These propagate at strongly oblique angles with respect to $\mathbf{B}_{\circ}$ (e.g., $\theta_{\mathrm{kb}} \sim 50^{\circ}$ ) toward upstream away from the shock. The two types have been labeled MTSI1 and MTSI2, respectively, by Matsukiyo and Scholer (2006). Note that the MTSI1 and MTSI2 labels stem just from the order at which they appeared in their simulations. In reference to their physical differences we label the instabilities in this paper as MTSI-SD (slow drift case) and MTSI-FD (fast drift case). It is important to note that when the instability is driven by the reflected ion beam (MTSI-FD), the resulting whistlers have a Poynting flux that is also upstream directed, the same as can occur for whistlers generated in the steepening ramp. 
Observations suggestive of MTSI-SD have been reported by Scudder, Mangeney, Lacombe, Harvey, Wu, et al. (1986) and Wilson et al. (2012). Adding to the puzzle, in a simulation study of the Lobzin et al. (2007) event, which was purported to support the dispersive radiation model, Comisel et al. (2011) presented another explanation. They proposed that the MTSI-SD, due to the slow drift of the incoming ion core, generated the whistlers, yet the waves were reflected toward upstream in the region of the overshoot, so that the observed upstream-propagating whistlers were not the signature of the model described by Krasnoselskikh et al. (2002). Based on a detailed analysis of wave vector orientations with respect to shock geometry and magnetic field, the study by Hull et al. (2012) presented arguments supportive of oblique whistlers due to the fast drift of the reflected ion beam (thus MTSI-FD). However, in that study emissions originating in the ramp via dispersive processes was not ruled out. The study by Dimmock et al. (2013) also presented oblique whistler observations from Cluster that have characteristics that could be related to shock macrodynamics; however, the MTSI-FD could not be ruled out.

Clearly more work needs to be done to understand source mechanisms of these whistlers. Reconciliation of the source mechanisms is important, as it is essential to understanding the nature of shock structure, stability, and dynamics, which can have dramatic consequences to plasma energization and thermalization. At shocks, whistler waves are well known to occur in the frequency range between the ion cyclotron frequency and the electron cyclotron frequency $\left(f_{\mathrm{ci}}<f_{\mathrm{wh}}<f_{\mathrm{ce}}\right)$. Where the waves lie in this range can have fundamentally different source mechanisms responsible for their generation. Thus, placing the observed wave frequencies into proper context with the characteristic plasma frequencies is an essential consideration in source mechanism identification. Comparisons with models therefore require accurate determinations of the wave properties, such as wave vector orientations, rest frame frequencies, and wavelengths, which are inherently difficult to determine within the layer of the shock. This is owing to the fact that under certain orientations and low frequencies, these waves can be significantly Doppler shifted in frequency in spacecraft frame measurements. This effect can be corrected for via multisatellite observations with spacing less than the characteristic wavelengths of the waves so that the waves are coherent and allow for timing analysis. Sufficiently resolved plasma measurements are also needed in order to determine plasma rest frame frequencies and characteristic parameters, and also to compare instability model predictions with features in the distribution. While the requisite observations have been available from the ISEE, AMPTE, and Cluster multispacecraft missions upstream of the ramp, where conditions are more steady, well-resolved plasma measurements within the layer of the shock have only recently been available from the Magnetospheric Multiscale (MMS) mission. This, coupled with high-time-resolution measurements of all components of the electric and magnetic fields on MMS, enables an unprecedented opportunity to accurately determine the properties of whistlers within the layer, assess sources, and evaluate consequences to shock structure and plasma transport.

In this paper we report the results of our analysis of whistler waves observed within the layer of a quasiperpendicular Earth bow shock event sampled by the MMS mission on 4 November 2015 at 0805:50 UT. While we seek to characterize whistler waves that occur over multiple frequencies/scales, particular attention is focused on the properties and consequences of intense obliquely propagating whistler waves near the lower-hybrid frequency regime. The intent of this work is to provide an important observational basis that can be used to test and constrain models for the generation of these waves. This event occurred under elevated solar wind flow conditions $\left(V_{\mathrm{sw}} \sim 710 \mathrm{~km} / \mathrm{s}\right)$ and thus was encountered somewhat closer to the Earth than usual (e.g., $\left.\mathbf{R}_{\mathrm{GSE}}=(11.1,4.2,-0.7) R_{E}\right)$. Such high solar wind flow conditions are conducive to more intense whistler waves near lower-hybrid frequencies. From a detailed characterization of the whistler waves, comparisons with the ion distribution features, we show that the source of these intense whistlers could be related to the MTSI-FD mechanism. We also show that these LH whistlers are affecting shock structure and plasma transport in a significant way, by inducing electron anisotropies and carrying sizable currents that lead to secondary higher-frequency whistler and electrostatic instabilities, respectively.

\section{Instrumentation and Experimental Data Set}

We used plasma data from the fast plasma investigation (FPI) for MMS (Pollock et al., 2016). In burst mode electron and ion distributions and moments are typically provided down to 30 and 150 mstime resolutions, respectively. We used magnetic field data from the fluxgate magnetometer (FGM) instrument (Russell et al., 2016) and also the search coil magnetometer instrument (SCM) (Le Contel et al., 2016). In burst mode all three magnetic field components are provided at a rate of $128 \mathrm{~S} / \mathrm{s}$ and at 8,192 S/s from FGM and SCM, 


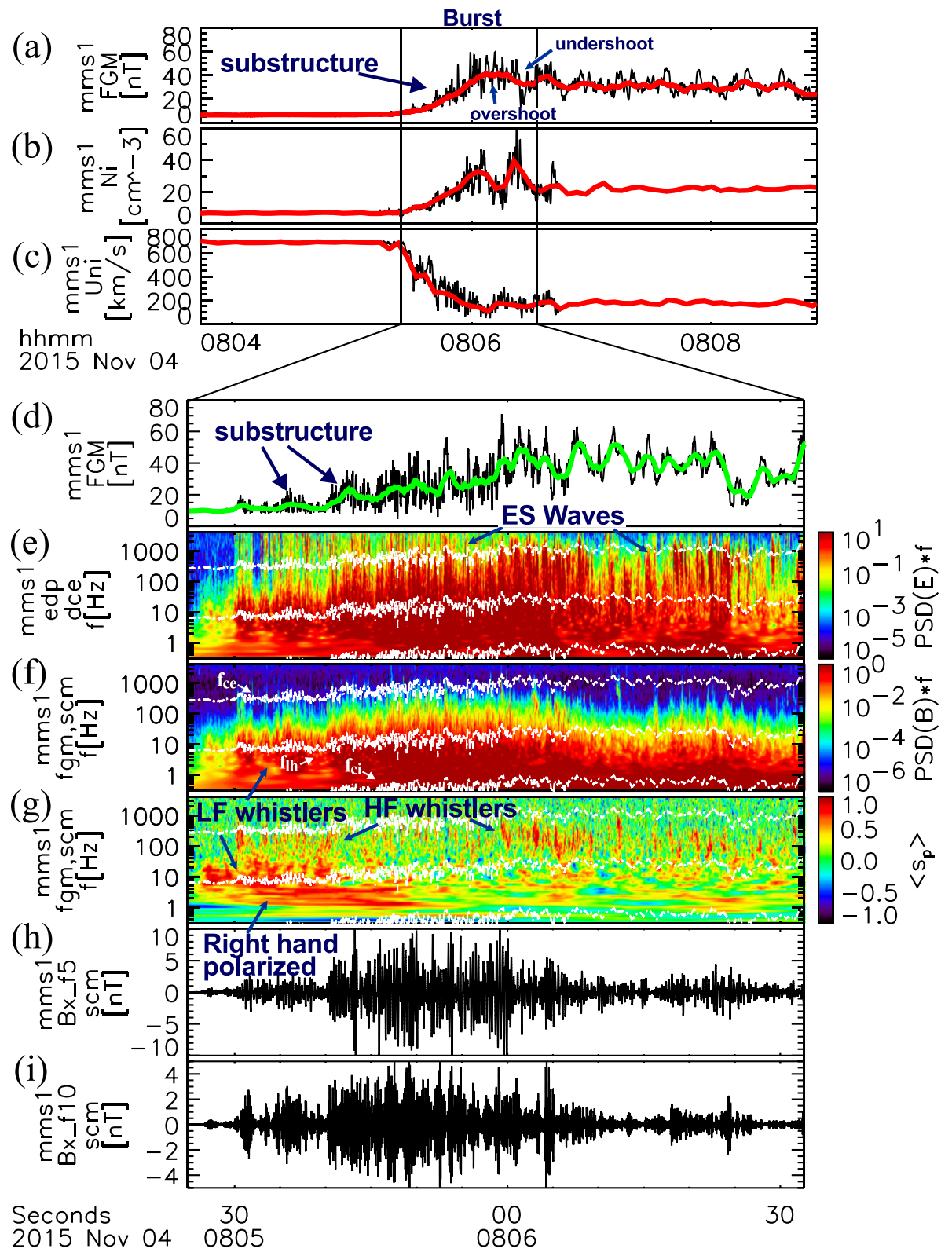

Figure 1. Shown are the (a) magnetic field amplitude, (b) ion density, and (c) NIF frame bulk speed along the shock normal. Vertical lines in Figures 1a-1c indicate the burst sampled interval. Also shown in the burst interval are the (d) magnetic field magnitude, the normalized power spectral densities of (e) the electric field, and (f) magnetic field from FGM and SCM, (g) magnetic field polarization, and GSE-x component of the magnetic field from SCM at (h) $5 \mathrm{~Hz}$ and (i) $10 \mathrm{~Hz}$.

respectively. Electric field data at 8,192 S/s used are from the axial and spin plane double-probe electric field sensors (ADP and SDP) (Ergun et al., 2016; Lindqvist et al., 2016).

\section{Observations}

\subsection{Overview}

Plasma and magnetic field data from one of the spacecraft (SC1) during the MMS inbound transect (upstream to downstream) of Earth's bow shock on November 042015 is shown in Figure 1. This is a quasiperpendicular bow shock transition, with the angle between the shock normal and upstream magnetic field estimated at $\theta_{\mathrm{bn} 1}^{\mathrm{RH}}=82^{\circ} \pm 2^{\circ}$. The upstream magnetic field vector is $\mathbf{B}_{1}=[-2.195,7.194,-1.482]$ and shock normal is estimated at $\hat{\mathbf{n}}_{\mathrm{RH}}=[0.9867,0.1615,-0.01916]$ via the Viñas and Scudder (1986) least 
squares Rankine-Hugoniot method. The velocity of the shock along the normal in the spacecraft frame is estimated at $v_{\mathrm{sh}}^{\mathrm{RH}}=25 \mathrm{~km} / \mathrm{s}$ from the RH method. The values for the RH determined normal and shock velocity were cross-checked with other methods and found to be consistent. We also verified that the magnetic field projected along the normal was reasonably constant, with no sharp excursions. These comparisons demonstrate that the normal is well determined in this case (see Appendix A1for a detailed description of the different estimates of the shock normal and relative velocities in addition to a demonstration of quasi-stationarity and characteristic asymptotic parameter determinations). The upstream Alfvén Mach number in the shock rest frame is estimated at $M_{A} \sim 10$ from a shock rest frame upstream normal flow of $U_{n 1}=\left|\mathbf{V s w} \cdot \hat{\mathbf{n}}_{\mathrm{RH}}-v_{\mathrm{sh}}^{\mathrm{RH}}\right|=|-710-25| \sim 735 \mathrm{~km} / \mathrm{s}$ and Alfvén speed of $72 \mathrm{~km} / \mathrm{s}$. The fast mode Mach number is estimated at $M_{f} \sim 6$ 6.7. This value was obtained from upstream plasma density, magnetic field, and electron and ion temperatures estimated at $N_{1}=6.5 \mathrm{~cm}^{-3}, B_{1}=8.4 \mathrm{nT}, T_{e 1}=14.3 \mathrm{eV}$, and $T_{i 1}=26$ $\mathrm{eV}$, respectively. Note that OMNIWeb proton temperatures from ACE mapped to the bow shock nose was used to estimate $T_{i 1}$ because upstream ion temperatures from FPI are overestimated, since it does not fully resolve the relatively cold solar wind ion distribution. This value is in reasonable agreement with an estimate based on the empirical temperature-velocity relation reported by Burlaga and Ogilvie (1973), which yielded a value of $35 \mathrm{eV}$, despite the flow for this shock being at or just above the upper limit of solar wind velocities used in the empirical relation. Based on this comparison, $M_{f}$ is confirmed to be a reasonable estimate.

An overview of the magnetic field, ion density, and magnitude of the ion bulk velocity along the normal in the shock normal incidence frame (NIF) is given in Figures 1a-1c, respectively. Characteristic of a fast mode shock transition, the magnetic field increases across the shock as the plasma gets compressed and slows down. The $12 \mathrm{~s}$ boxcar averaged magnetic field profile indicated in red in Figure 1a shows the standard foot, ramp, overshoot, and undershoot features that are characteristic of a supercritical shock transition. The overall thickness of the shock from the start of the foot to the overshoot region (0805:21 to 0806:09 UT) is estimated at $L_{\mathrm{sh}} \sim 1,200 \mathrm{~km}=1.3 L_{\text {ciil1 }}=13 \lambda_{\mathrm{i} 1}$, where $L_{\text {ciil1 }}=U_{\mathrm{n} 1} /\left(2 \pi f_{\mathrm{ci}}\right)$ is the upstream convected ion inertial length scale and $\lambda_{\mathrm{i} 1}$ the upstream ion inertial length, respectively. At higher resolution, the magnetic ramp has quasiperiodic substructure that increases in strength with increasing penetration in the shock (black curve in Figure 1a). Similar variations are seen in the burst resolution ion density and normal bulk speed. Vertical lines in Figures $1 \mathrm{a}-1 \mathrm{c}$ indicate a region where the data are sampled in burst mode. The region shown encompasses the foot, ramp, overshoot, and undershoot region of the shock (see magnetic field profile in Figure 1a).

To highlight the multiscale nature of the layer, Figure 1d provides the burst-sampled magnetic field from FGM (black curve) and the $1 \mathrm{~s}$ boxcar-averaged magnetic field (green curve) in the burst interval. The averaged magnetic field profile makes apparent the substructure enhancements, which occur at a cadence of 3-4 s. The burst magnetic field data reveals the presence of large-amplitude shorter-duration magnetic structure superposed on the magnetic substructure. To assess the nature of these shorter-scale features, Figures 1e and 1f show dynamic electric and magnetic field (from merged FGM and SCM data) power spectral densities (PSDs) that have been multiplied by frequency to make apparent structure at higher frequency. To construct the PSDs, a Morlet wavelet transform is applied to time series data. By construction, the resulting power spectra with proper normalization yields meaningful PSD estimates (Eriksson, 1998). Within a constant factor, the rescaled PSDs in Figures 1e and 1f give the relative contribution to the total integrated power at a given time (e.g., $P^{\operatorname{tot}}(t)=\Delta x \sum P S D(f, t) f$, where the spacing $\Delta x=\Delta f / f$ is by construction a fixed constant). The normalized PSDs reveal that the finer-scale magnetic signatures are due to intense bursts of electromagnetic (EM) waves, which are indicated by the appearance of similar electric and magnetic signatures at frequencies below a few hundred Hertz. The EM waves span the frequency range between the ion cyclotron (white curve near a few tenths of $\mathrm{Hz}$ ) and the electron cyclotron frequencies (white curve above hundred $\mathrm{Hz}$ ), which corresponds to the whistler mode regime. The EM waves are more intense near and below the lower-hybrid frequency (indicated by white curve near $10 \mathrm{~Hz}$ in Figures 1e and 1f). These low-frequency (LF) EM waves are shown to be right-hand polarized in the first part of the interval from 0805:25 UT to 0805:50 UT in Figure 1g, which indicates that these are whistler mode waves. Note, red (blue) in Figure $1 \mathrm{~g}$ corresponds to right-hand (left-hand) polarization. At later times, the LF whistler polarization is less discernible, owing to a combination of effects, such as Doppler effects and/or mixtures of modes in a given frequency band. Example, magnetic field waveforms at 5 and $10 \mathrm{~Hz}$ from SCM show these appear as bursts of wave activity that increase in intensity with further penetration into the ramp (see Figures $1 \mathrm{~h}$ and 1i). These intense bursts of whistler activity are generally collocated with the magnetic ramp substructure 
enhancements. Less intense higher-frequency (HF) whistlers at a few hundred Hertz also occur throughout the interval. The presence of HF whistlers is readily seen by their bursty right-hand polarization (red) signatures approaching the electron cyclotron frequency in Figure 1g. Similar observations were reported by Hull et al. (2012), who showed evidence indicating that the HF whistlers are causally connected to the LF whistlers. Strong electrostatic (ES) waves are also present throughout the interval, as indicated by the broadband activity near and well above $100 \mathrm{~Hz}$ in Figure 1e, with no magnetic field counterpart seen in Figure 1f. Interestingly, the strong ES waves have a cadence near the LH whistler frequency suggesting that their excitation/structuring are causally related to the LF whistlers. The association of LF whistlers with the HF whistlers and the ES waves suggests that the LF whistlers are influencing the shock structure and interacting with the plasma.

\subsection{Wave Vectors, Phase Speeds, and Wavelengths}

MMS enables accurate determinations of the wave vector, phase speeds, and rest frame frequencies, which are necessary for distinguishing source mechanisms. We used two independent methods to determine the wave vectors. One method used is the four-spacecraft time-of-flight- (TOF-)based analysis method applied to pass-filtered magnetic field data (Harvey, 1998). This method provides accurate estimates of $\hat{\mathbf{k}}$, the phase speed, and wavelength provided that the waveform is suitably stationary and that timings are well resolved from the sampling resolution. To corroborate timing results, we also used minimum variance analysis (MVA) method applied to magnetic field measurements. While this could be done spectrally via the Means (1972) method, it is useful for direct comparisons with other methods to do this analysis with pass-filtered time domain signals. This approach yields wave vector directions to within a $180^{\circ}$ ambiguity, which is removable with the aid of simultaneously measured 3-D electric fields and Faraday's Law. Use of Faraday's Law requires the electric field to be well measured on all baselines (including accurate determinations of shorting factors, which may be context dependent) and that there are no sizable electrostatic waves that can distort the whistler electric field signal.

Figure 2 shows an example interval of data in the foot region, where timing analysis was successfully applied. Figure 2a shows burst-sampled magnetic field data from the fluxgate magnetometer from each of the spacecraft, indicating the presence of strong magnetic field variations superposed on the background shock magnetic field. The normalized electric and magnetic field (from SCM) PSDs given in Figures $2 \mathrm{~b}$ and $2 \mathrm{c}$ show the presence of whistler waves at frequencies ranging from a few to $20 \mathrm{~Hz}$ (i.e., near and below $f_{\mathrm{lh}}$ ). Note, the reduction of intensity at frequencies $\lesssim 1 \mathrm{~Hz}$ in Figure $2 \mathrm{c}$ is artificial owing to the reduced sensitivity of the SCM at these lower frequencies and a high-pass filtering at $1 \mathrm{~Hz}$ applied to produce burst SCM L2 data.

Figures $2 \mathrm{~d}$ and $2 \mathrm{e}$ show that these are obliquely propagating and right-hand polarized. Note, the angles, determined via the Means method, in Figure $2 \mathrm{~d}$ have a $180^{\circ}$ ambiguity. Figures $2 \mathrm{f}-2 \mathrm{~h}$ show $10 \mathrm{~Hz}$ pass-filtered components of the magnetic field measured by SCM in GSE coordinates $B_{x}, B_{y}$, and $B_{z}$ from the four spacecraft. Similar time-delayed waveforms are apparent in the interval. Vertical lines delimit the region where cross-correlation analysis was applied to all possible spacecraft pairs of the $B_{x}$ traces to determine the delay times associated with an isolated packet. Peak correlation coefficients were found to have values $\geq 0.95$. The delay times $\left(\delta t_{\mathrm{ij}}=t_{i}-t_{j}\right)$ are found to be $\delta t_{12}=-1.83 \mathrm{~ms}, \delta t_{13}=-15.0 \mathrm{~ms}, \delta t_{14}=14.4 \mathrm{~ms}, \delta t_{23}=-13.4$ $\mathrm{ms}, \delta t_{24}=16.2 \mathrm{~ms}$, and $\delta t_{34}=30.0 \mathrm{~ms}$, which are well resolved from the SCM instrument sampling period of $0.122 \mathrm{~ms}$ in burst mode. Figures $2 \mathrm{i}-2 \mathrm{k}$ show the same three sets of profiles after shifting to MMS1's timeline using the appropriate delay time. The good alignment of the traces verifies that the delay times are well determined and that the $10 \mathrm{~Hz}$ whistler waveform is quasi-stationary over the spacecraft transit time. Given the delay times, the wave vector direction was found to be $\hat{\mathbf{k}}_{\text {tof }}=[0.730,0.024,0.683]$ in GSE coordinates via the Harvey (1998) method, which is outlined in Appendix A1. The wave packet was propagating in the upstream direction at an angle with respect to the shock normal $\theta_{\mathrm{kn}}=45^{\circ}$, and oblique to the magnetic field with $\theta_{\mathrm{kb}}=126^{\circ}$. The phase speed in the spacecraft frame was found to be $V_{\mathrm{psc}}=447 \mathrm{~km} / \mathrm{s}$. In the shock frame this corresponds to an apparent phase speed along the normal estimated at $V_{\mathrm{pn}}^{\mathrm{sh}}=V_{\mathrm{psc}} / \cos \theta_{\mathrm{kn}}-v_{\mathrm{sh}}=607 \mathrm{~km} / \mathrm{s}$, where $v_{\mathrm{sh}}=25 \mathrm{~km} / \mathrm{s}$ is the shock velocity in the spacecraft frame determined from the RH method. Thus, the wave packet is not a phase standing feature of the shock, but propagates upstream of the shock against the incoming solar wind flow. Given the locally measured center of mass velocity $\mathbf{V}_{\mathrm{cm}}=[-494,150,218] \mathrm{km} / \mathrm{s}$, the phase speed in the plasma rest frame is found to be $V_{p}=V_{\mathrm{psc}}-\mathbf{V}_{\mathrm{cm}} \cdot \hat{\mathbf{k}}_{\mathrm{tof}}=655 \mathrm{~km} / \mathrm{s}$. The wavelength is $\lambda \equiv V_{\mathrm{psc}} / f_{\mathrm{sc}}=44.7 \mathrm{~km}=0.5 \lambda_{i}$, where $\lambda_{i} \equiv c / \omega_{\mathrm{pi}}$ is the local ion inertial length and $f_{\mathrm{sc}}$ is the spacecraft frame 


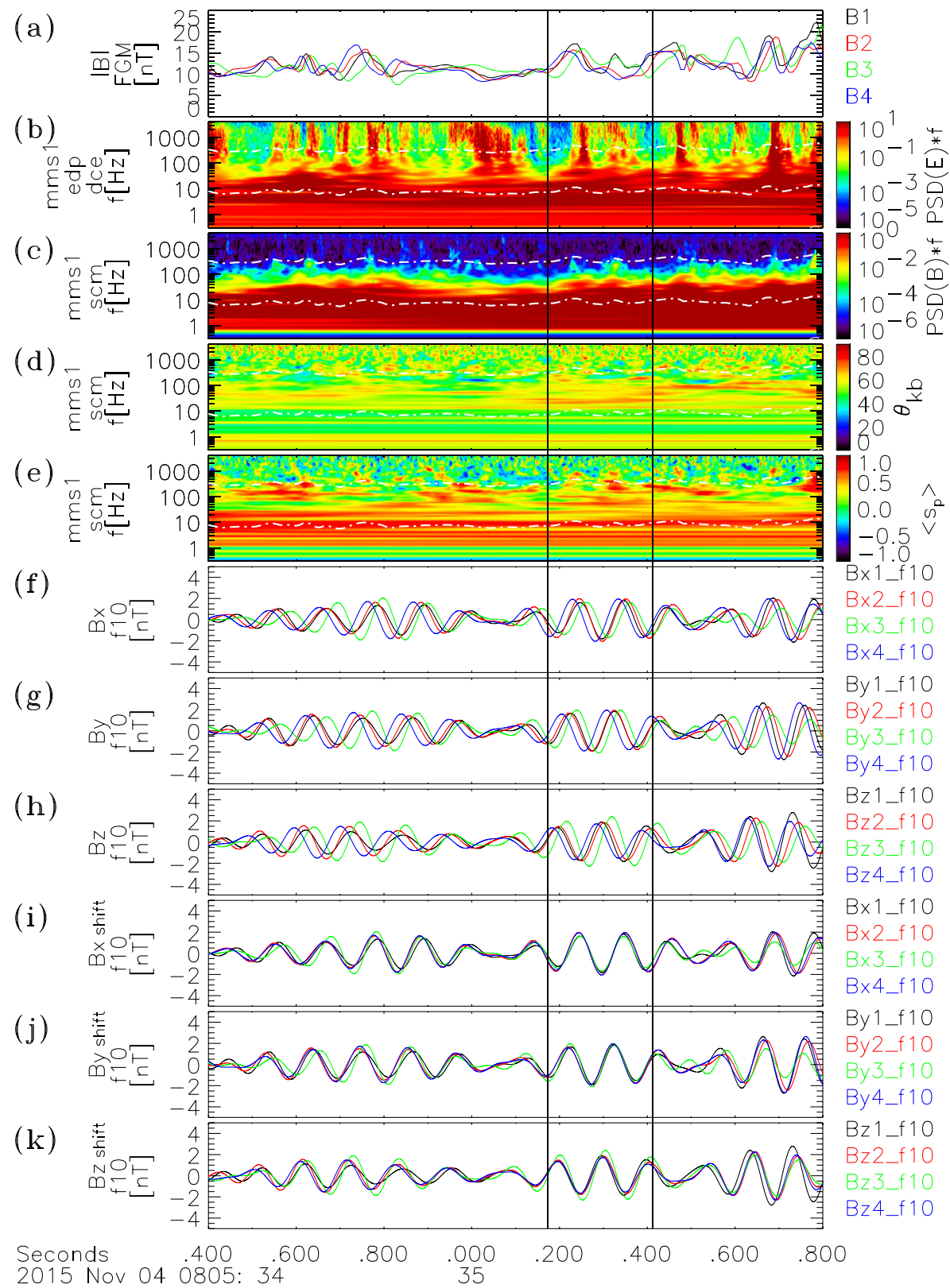

Figure 2. Shown are the (a) magnetic field magnitude from all four SC, the normalized PSD of the (b) electric field, and (c) magnetic field from SCM, (d) the wave-normal angle with respect to B determined by the Means method, (e) polarization, components of the magnetic field (f) $B_{x}$, (g) $B_{y}$, and (h) $B_{z}$ in GSE and pass-filtered at $10 \mathrm{~Hz}$ for all SC. Time-shifted $10 \mathrm{~Hz}$ pass-filtered components of the magnetic field (i) $B_{x \text { shift }}$, (j) $B_{y \text { shift }}$, and (k) $B_{z \text { shift }}$ in for all SC.

frequency, which in this case is $10 \mathrm{~Hz}$. After correcting for the Doppler effect, the rest frame frequency is $f_{\mathrm{o}}=f_{\mathrm{sc}}-\mathbf{V}_{\mathrm{cm}} \cdot \hat{\mathbf{k}}_{\mathrm{tof}} / \lambda=14.7 \mathrm{~Hz}=1.9 f_{\mathrm{lh}}$.

As a comparison, wave unit vectors in GSE coordinates from each spacecraft estimated by the MVA method constrained via Faraday's law are found to be $\hat{\mathbf{k}}_{\mathrm{mv1}}=[0.720,0.043,0.693], \hat{\mathbf{k}}_{\mathrm{mv} 2}=[0.659,0.077,0.748]$, $\hat{\mathbf{k}}_{\mathrm{mv} 3}=[0.736,-0.033,0.676]$, and $\hat{\mathbf{k}}_{\mathrm{mv} 4}=[0.642,0.036,0.766]$. These values are reasonably close to the timing results, with respective angles between TOF and MVA methods determined to be $\cos ^{-1}\left(\hat{\mathbf{k}}_{\text {tof }} \cdot \hat{\mathbf{k}}_{\text {mv1 }}\right)=1.3^{\circ}$, $\cos ^{-1}\left(\hat{\mathbf{k}}_{\text {tof }} \cdot \hat{\mathbf{k}}_{\mathrm{mv2}}\right)=6^{\circ}, \cos ^{-1}\left(\hat{\mathbf{k}}_{\text {tof }} \cdot \hat{\mathbf{k}}_{\mathrm{mv} 3}\right)=3^{\circ}$, and $\cos ^{-1}\left(\hat{\mathbf{k}}_{\text {tof }} \cdot \hat{\mathbf{k}}_{\mathrm{mv} 4}\right)=7^{\circ}$. The close agreement between the TOF and MVA results show that the wave vector direction is well-determined for this case.

To verify the isolated waveform is a whistler wave, hodograms of the magnetic fields sampled by the four spacecraft within the interval are given in Figure 3. The first column compares the two magnetic field 


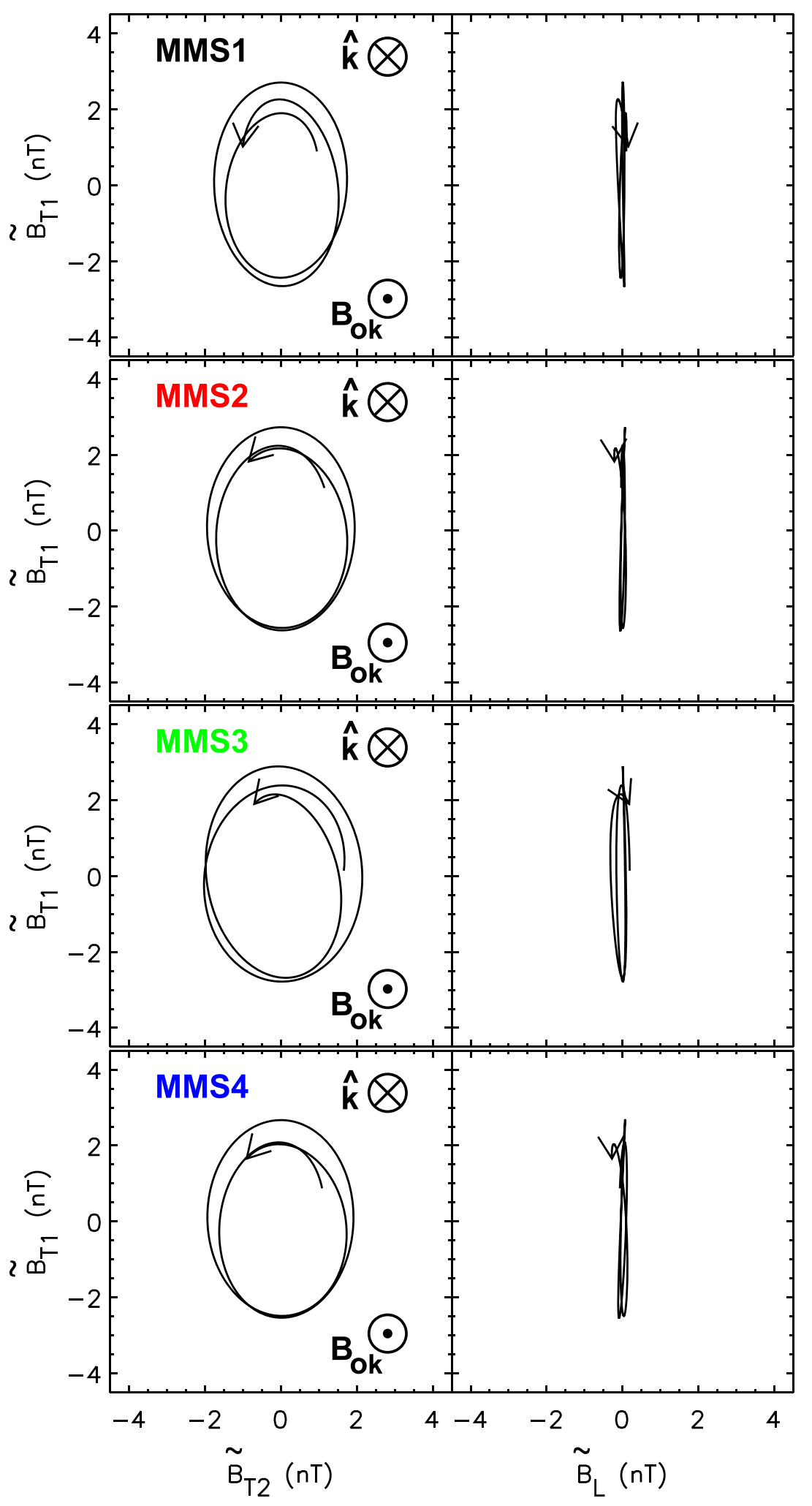

Figure 3. Shown are hodograms of the magnetic field sampled by each spacecraft within the interval depicted in Figure 2. Also shown in the first column is the wave vector direction $\hat{\mathbf{k}}$ and the sense of the projection of the background magnetic field $\mathrm{B}_{\mathrm{o} k}$ along the wave vector. 
Table 1

Waveform Parameters

\begin{tabular}{|c|c|c|c|c|c|c|c|c|c|c|c|c|c|}
\hline Time & $f_{\mathrm{sc}}(\mathrm{Hz})$ & $f_{\circ}(\mathrm{Hz})$ & $f_{\circ} / f_{l h}$ & $\lambda(\mathrm{km})$ & $V_{p}(\mathrm{~km} / \mathrm{s})$ & $V_{p} / V_{A}$ & $\lambda / \lambda_{i}$ & $\hat{\mathbf{k}}$ & $\mathbf{B}_{\circ}(\mathrm{nT})$ & $\theta_{\mathrm{kb}}$ & $\theta_{\mathrm{kn}}$ & $V_{\mathrm{pn}}^{s h}(\mathrm{~km} / \mathrm{s})$ & $V_{\text {pth }}(\mathrm{km} / \mathrm{s})$ \\
\hline 0805:30.930 & 15 & 22.9 & 2.7 & 27.3 & 624 & 6.3 & 0.34 & {$[0.78,-0.01,0.63]$} & {$[-3.0,11.2,-5.3]$} & $117^{\circ}$ & $41^{\circ}$ & 517 & 665 \\
\hline 0805:31.533 & 5 & 11.7 & 1.5 & 44.0 & 514 & 5.4 & 0.53 & {$[0.84,-0.18,0.52]$} & {$[-2.2,10.5,-4.9]$} & $122^{\circ}$ & $38^{\circ}$ & 255 & 537 \\
\hline 0805:31.580 & 10 & 12.6 & 1.7 & 44.0 & 553 & 6.1 & 0.54 & {$[0.64,-0.15,0.75]$} & {$[-2.1,10.4,-4.6]$} & $123^{\circ}$ & $53^{\circ}$ & 706 & 554 \\
\hline 0805:33.338 & 5 & 12.5 & 1.8 & 45.6 & 571 & 6.5 & 0.54 & {$[0.87,-0.09,0.48]$} & {$[-2.2,8.7,-6.0]$} & $121^{\circ}$ & $33^{\circ}$ & 247 & 532 \\
\hline 0805:33.338 & 10 & 16.8 & 2.3 & 37.1 & 622 & 6.9 & 0.44 & {$[0.76,-0.09,0.64]$} & {$[-2.3,8.7,-6.3]$} & $127^{\circ}$ & $44^{\circ}$ & 491 & 665 \\
\hline 0805:33.338 & 15 & 21.2 & 3.0 & 31.6 & 669 & 5.3 & 0.37 & {$[0.70,-0.02,0.71]$} & {$[-2.2,8.7,-6.0]$} & $124^{\circ}$ & $48^{\circ}$ & 683 & 698 \\
\hline 0805:34.646 & 15 & 16.5 & 2.3 & 31.5 & 642 & 7.2 & 0.38 & {$[0.53,0.09,0.84]$} & {$[-2.5,9.7,-4.8]$} & $113^{\circ}$ & $59^{\circ}$ & 1106 & 521 \\
\hline 0805:35.293 & 10 & 14.7 & 1.9 & 44.7 & 655 & 7.1 & 0.54 & {$[0.73,0.02,0.68]$} & {$[-2.6,8.5,-7.5]$} & $126^{\circ}$ & $45^{\circ}$ & 607 & 615 \\
\hline 0805:35.397 & 10 & 14.2 & 1.8 & 43.7 & 622 & 6.6 & 0.54 & {$[0.76,0.15,0.63]$} & {$[-2.8,8.8,-7.9]$} & $119^{\circ}$ & $40^{\circ}$ & 546 & 545 \\
\hline 0805:35.906 & 15 & 13.5 & 1.5 & 44.0 & 592 & 6.1 & 0.59 & {$[0.46,0.21,0.86]$} & {$[-2.8,9.5,-9.4]$} & $123^{\circ}$ & $62^{\circ}$ & 1380 & 559 \\
\hline 0805:37.832 & 10 & 14.0 & 1.9 & 45.8 & 642 & 6.8 & 0.53 & {$[0.59,0.16,0.79]$} & {$[-2.9,7.5,-8.2]$} & $128^{\circ}$ & $54^{\circ}$ & 754 & 647 \\
\hline 0805:39.167 & 10 & 17.9 & 2.4 & 39.7 & 713 & 7.7 & 0.45 & {$[0.72,-0.14,0.68]$} & {$[-1.2,9.4,-5.7]$} & $123^{\circ}$ & $48^{\circ}$ & 569 & 671 \\
\hline 0805:41.091 & 10 & 11.3 & 1.2 & 40.9 & 463 & 4.4 & 0.54 & {$[0.55,-0.20,0.81]$} & {$[-1.7,12.4,-7.5]$} & $130^{\circ}$ & $60^{\circ}$ & 793 & 595 \\
\hline 0805:41.816 & 15 & 11.9 & 1.0 & 39.0 & 463 & 3.8 & 0.58 & {$[0.56,0.28,0.78]$} & {$[-2.9,16.9,-8.1]$} & $100^{\circ}$ & $55^{\circ}$ & 995 & 301 \\
\hline 0805:41.902 & 5 & 4.5 & 0.4 & 73.0 & 328 & 2.8 & 1.1 & {$[0.59,-0.03,0.81]$} & {$[-2.3,17.8,-6.9]$} & $113^{\circ}$ & $56^{\circ}$ & 628 & 291 \\
\hline 0805:43.205 & 5 & 5.2 & 0.4 & 78.5 & 406 & 3.8 & 1.3 & {$[0.68,0.12,0.72]$} & {$[-5.4,15.4,-7.5]$} & $114^{\circ}$ & $47^{\circ}$ & 550. & 297 \\
\hline 0805:47.919 & 5 & 8.8 & 0.7 & 39.5 & 347 & 2.9 & 0.63 & {$[0.70,-0.11,0.71]$} & {$[-0.7,14.3,-13.8]$} & $126^{\circ}$ & $49^{\circ}$ & 276 & 494 \\
\hline
\end{tabular}

components transverse to the wave vector for each of the spacecraft. In the first column the wave vector points into the page, while the projection of the background magnetic field along the wave vector points out of the page. The second column compares the longitudinal component $\tilde{B}_{L}$, which is along the wave vector direction, with the transverse component $\tilde{B}_{T 1}$, which is along the maximum variance direction. The hodograms show the characteristic feature expected for oblique whistler waves propagating antiparallel to the background magnetic field. Namely, the wave magnetic field is right-hand elliptically polarized with respect to the background magnetic field in the plane transverse to the wave vector. The close similarity in the hodograms verifies the quasistationary character of the whistler waveform.

Table 1 lists characteristic parameters of 17 whistler waveforms at frequencies from 5 up to $15 \mathrm{~Hz}$ that were suitably stationary for accurate determinations of wave vectors and phase speeds using the TOF approach. This list, which is not meant to be exhaustive, is a representative sampling at key locations with different density, magnetic field, and associated ion properties for detailed comparisons with source mechanisms of whistlers near lower-hybrid frequencies to be discussed in section 3.5. These whistlers have rest frame frequencies relatively near the lower-hybrid frequency $\left(f_{\circ} / f_{\mathrm{lh}}\right.$ ranging from 0.4 to 3$)$ and wavelengths $\lambda$ ranging from $\sim 0.3$ to $\sim 1.3 \lambda_{i}$ (or $\sim 14.5$ to $\sim 53.9 \lambda_{e}$, where $\lambda_{\mathrm{e}} \equiv c / \omega_{\mathrm{pe}}$ ). To situate these wavelengths in terms of the whistler dispersion, we remind the reader that the dispersion curve of oblique whistlers $\omega(\mathrm{k})$ at fixed $\theta_{\mathrm{bk}}$ presents a double curvature (e.g., Swanson, 1989, Figure 3.1). It is first concave with the group speed increasing with $k$. At $k \lambda_{\mathrm{e}} \approx 0.58$ there is an inflection point beyond which the curve becomes concave and saturates for large wave numbers. The measured whistlers, having $k \lambda_{e}$ ranging from 0.12 to 0.43 , are thus situated below the inflection point where the curve is still concave, hence the group velocity increases with $k$.

The waveforms are not phase-standing features of the shock, but propagate upstream $\left(\theta_{\mathrm{kn}}<90^{\circ}\right)$ at oblique angles antiparallel to the ambient magnetic field (see Table 1 eleventh column). Though there are a few examples inclined toward the normal $\left(\theta_{\mathrm{kn}}<45^{\circ}\right)$, the majority of the waveforms have wave vectors that are oriented at quite an oblique angle to the normal (with $\theta_{\mathrm{kn}}>45^{\circ}$ for 11 out of 17 events in column 12 of Table 1). The plasma frame phase speeds $V_{p}$ range from a few hundred to several hundred $\mathrm{km} / \mathrm{s}$, corresponding to 2.8 to $7.7 V_{A}$, where $V_{A}$ is the local Alfvén speed (sixth and seventh columns of Table 1). These values correspond to phase speeds projected along the normal in the shock frame $V_{\mathrm{pn}}^{\mathrm{sh}}$ that range from few hundreds to nearly $1,400 \mathrm{~km} / \mathrm{s}$.

To compare, a theoretical estimate for the phase speed can be obtained from the dispersion relation for low-frequency electromagnetic waves in a cold plasma (Stringer, 1963). Assuming that the phase speed is 
much larger than the acoustic speed, one can write (Hull et al., 2012)

$$
V_{\mathrm{pth}}\left(f, \theta_{\mathrm{kb}}\right)=V_{A}\left[\left(\frac{m_{i}}{m_{e}} \cos ^{2} \theta_{\mathrm{kb}}\left(\frac{f}{f_{\mathrm{lh}}}\right)^{2}+\frac{\sin ^{4} \theta_{\mathrm{kb}}}{4}\right)^{1 / 2}+1-\frac{\sin ^{2} \theta_{\mathrm{kb}}}{2}-\left(\frac{f}{f_{\mathrm{lh}}}\right)^{2}\right]^{1 / 2}
$$

Here $m_{i} / m_{e}$ is the ion to electron mass ratio. In the first parenthesis the term with the mass ratio largely dominates the $\sin ^{4} \theta_{\mathrm{kb}}$ term for frequencies in the lower-hybrid range and above, unless the propagation is strictly perpendicular. Since we have determined that $\theta_{\mathrm{kb}} \neq 90^{\circ}$, the equation can be simplified to

$$
V_{\mathrm{pth}}\left(f, \theta_{\mathrm{kb}}\right)=V_{A}\left[\frac{f}{f_{\mathrm{lh}}}\left(\left(\frac{m_{i}}{m_{e}}\right)^{1 / 2} \cos \theta_{\mathrm{kb}}-\frac{f}{f_{\mathrm{lh}}}\right)+1-\frac{\sin ^{2} \theta_{\mathrm{kb}}}{2}\right]^{1 / 2}
$$

One can see that the phase speed increases with frequency when it is near $f_{\mathrm{lh}}$ and maximizes for $f^{*}=$ $0.5 \cos \theta_{\mathrm{kb}} f_{\mathrm{ce}}$, where it reaches the value $V_{\mathrm{pth}}^{*} \approx 0.5 \sqrt{m_{i} / m_{e}} \cos \theta_{\mathrm{kb}} V_{A}$ at the short wavelength $\lambda^{*}=c / f_{\mathrm{pe}}$. The phase speeds $V_{\text {pth }}$ computed via equation (2) are given in the last column of Table 1 . The values for $V_{\text {pth }}$ are in reasonably good quantitative agreement with the timing estimates $V_{p}$ given in the sixth column of Table 1 (better than $20 \%$ for majority of events, with a few exceptions approaching $30 \%$ to $40 \%$ ). The good agreement indicates that the wave cone angles are well determined, that Doppler corrections are accurate, and that cold plasma theory provides a reasonable estimate of the phase speed (and also wavelengths) of the whistler waves occurring near lower hybrid frequencies.

\subsection{Currents}

The presence of intense whistler waves can significantly modify the shock magnetic field and hence the current distribution within the layer. To show how much is attributed to the LF whistler waves, Figure 4 shows current densities in a zoomed-in interval of the foot, bounding the third green substructure enhancement from the left of Figure 1d. The magnetic field magnitude is provided in Figure $4 \mathrm{a}$ for context and shows that the whistlers (which are near and below $10 \mathrm{~Hz}$ ) result in strong variations in the background field $\left(\delta B / B_{\circ} \sim 0.2-1.0\right)$. Current densities associated with the whistlers were determined using two methods. The first method used was the curlometer technique applied to burst-sampled fluxgate magnetometer data (e.g., Dunlop et al., 1988, 2002; Robert et al., 1998). This four-spacecraft approach is based on Ampere's law $\mathbf{J}_{\mathrm{url}}=\nabla \times \mathbf{B} / \mu_{\circ}$ and is accurate provided that the current structure is sufficiently large and time stationary over the spacecraft separations. The current density magnitude $\left|\mathbf{J}_{\text {curl }}\right|$ determined from the curlometer method is indicated by the black curve in Figure $4 \mathrm{~b}$. We also computed the current densities directly from the density and electron and ion bulk velocities: $\mathbf{J}_{\text {mom }}=e N\left(\mathbf{U}_{\mathbf{i}}-\mathbf{U}_{\mathbf{e}}\right)$. It is important to note that ion moments are sampled at a slower rate (150 ms time resolution) than the electrons (30 ms). To study structure at the highest time resolution possible, ion bulk velocities were matched to electron samples using linear interpolation. Such an approximation is justified, since the ions respond to changes in fields more slowly than the electrons. Current density magnitudes computed from relative ion-electron drifts from SC1 ( $\left.\left|\mathbf{J}_{\mathrm{mom} 1}\right|\right)$ and SC2 $\left(\left|\mathbf{J}_{\text {mom } 2}\right|\right)$ are shown in Figure $4 \mathrm{~b}$ as the red and green curves, respectively. The close similarity between the traces shows that the current densities were accurately determined and were quasistationary over the spacecraft fleet transit time with separations that ranged from $\sim 11 \mathrm{~km}$ to $18 \mathrm{~km}$ in extent. The $\left|\mathbf{J}_{\text {mom1 }}\right|$ and $\left|\mathbf{J}_{\text {mom } 2}\right|$ traces do show alternating time shifts indicating that the order which these spacecraft encounter a given current enhancement is changing. This appears to be attributed to slight changes/rotation in the propagation direction of whistler wave packets in the interval (not shown), which may be a spatial and/or a temporal effect. For context, Figure 4c displays the parallel (red) and perpendicular (blue) components of the currents from moments sampled on SC1. The currents are shown to be composed of admixtures of perpendicular and parallel contributions. The currents in Figures $4 \mathrm{~b}$ and $4 \mathrm{c}$ do show strong variations at frequencies from $10 \mathrm{~Hz}$ and below with peak amplitudes approaching $\sim 1-2 \mu \mathrm{A} / \mathrm{m}^{2}$ in the interval. These strong localized currents are attributed to the LF whistlers occurring in the interval. To verify this, Figure $4 \mathrm{~d}$ depicts the PSD of $\left|\mathbf{J}_{\text {mom } 1}\right|$, which clearly shows that important contributions are coming from fluctuations (whistlers) at $10 \mathrm{~Hz}$ and below. Close inspection of magnetic field waveforms indicates that regions of concentrated currents are due to notable differences in whistler wave packet signatures (orientation and magnitude) sampled by the different spacecraft. The peak amplitudes of the current in the foot associated with the whistlers are significantly larger than the background current estimated at $0.05 \pm 0.01 \mu \mathrm{Amp} / \mathrm{m}^{2}$ in the interval shown and also the background current in the steepest part of the ramp which peaks at $0.4 \pm 0.4 \mu \mathrm{Amp} / \mathrm{m}^{2}$ 
(a)

(b)

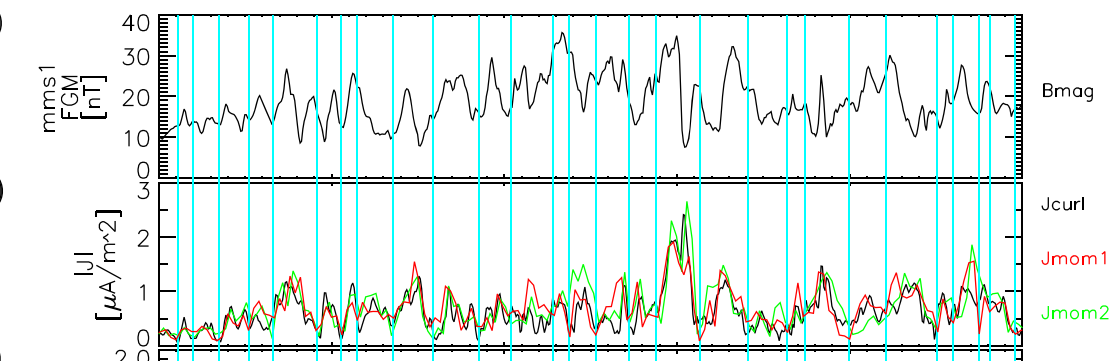

(c)

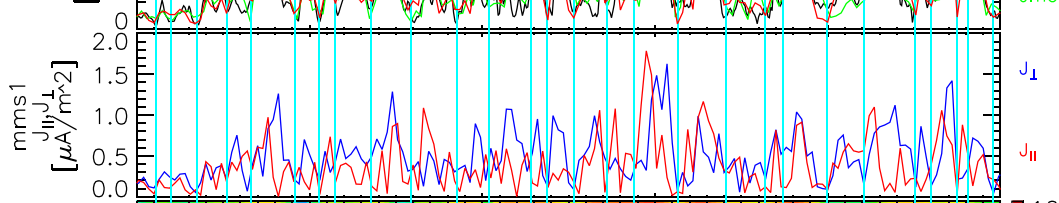

(d)

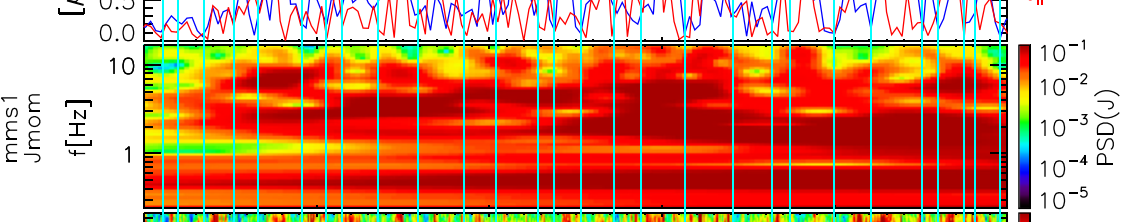

(e)
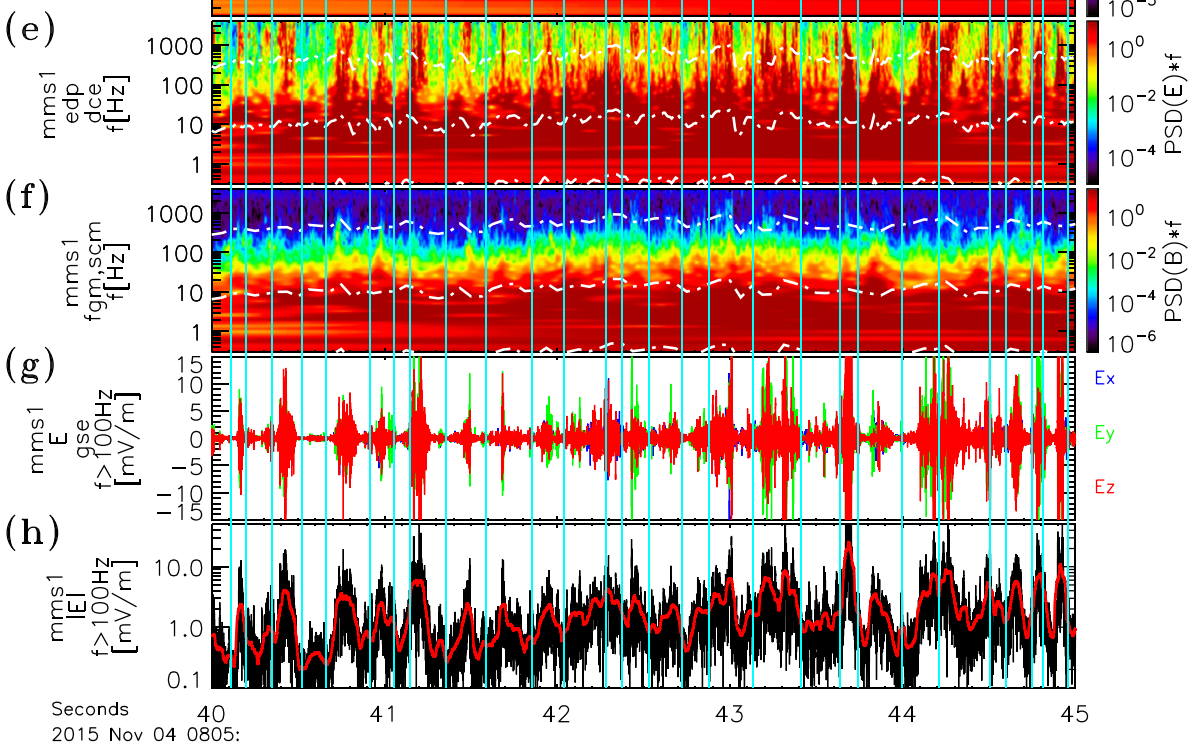

Figure 4. (a) Magnitude of magnetic field from FGM, (b) current density magnitude determined from the curlometer method (black), and moments from SC1 (red) and SC2 (green), (c) parallel and perpendicular components of the current based on moments from SC1, (d) PSD of current density from SC1 moments, the normalized PSD of the (e) electric field and (f) magnetic field from merged FGM and SCM data, (g) the electric field waveform above $100 \mathrm{~Hz}$ and (h) magnitude of the electric field waveform above $100 \mathrm{~Hz}$ with burst sampling in black and $30 \mathrm{~ms}$ boxcar averages in red. Fiducial vertical lines (cyan) delimit select adjacent intervals of current density maxima, as a visual aid.

(not shown). Note, background current values were estimated using the curlometer method applied to the background magnetic field depicted in Figure 1a. Moreover, the currents attributed to the whistlers approach a significant fraction of the electron thermal current with $\left|J / J_{\mathrm{vte}}\right| \sim 0.1-0.5$, where the thermal current is defined as $J_{\mathrm{vte}} \equiv e N V_{\mathrm{te}}$, with $V_{\mathrm{te}} \equiv \sqrt{k_{B} T_{e} / m_{e}}$ being the electron thermal speed. As a comparison with past observations, the currents estimated for the macroscale structure of a moderately strong supercritical shock reported by Scudder, Mangeney, Lacombe, Harvey, Aggson, et al. (1986) reached values up to 0.01 $\mu \mathrm{Amp} / \mathrm{m}^{2}$ in the steepest part of the ramp, which is much smaller than the values reported here. However, the whistler-associated currents are in line with the modest values of current estimated from shorter time scale magnetic field variations due to whistlers at $10 \mathrm{~Hz}$ and below reported by Wilson et al. (2014a, 2014b). Note that all of these past studies estimated currents from magnetic field gradients under the assumption that they were frozen to the shock. While such an approximation is valid for the shock macroscopic structure and phase standing whistlers, it is not valid for whistlers considered here, since they have been 


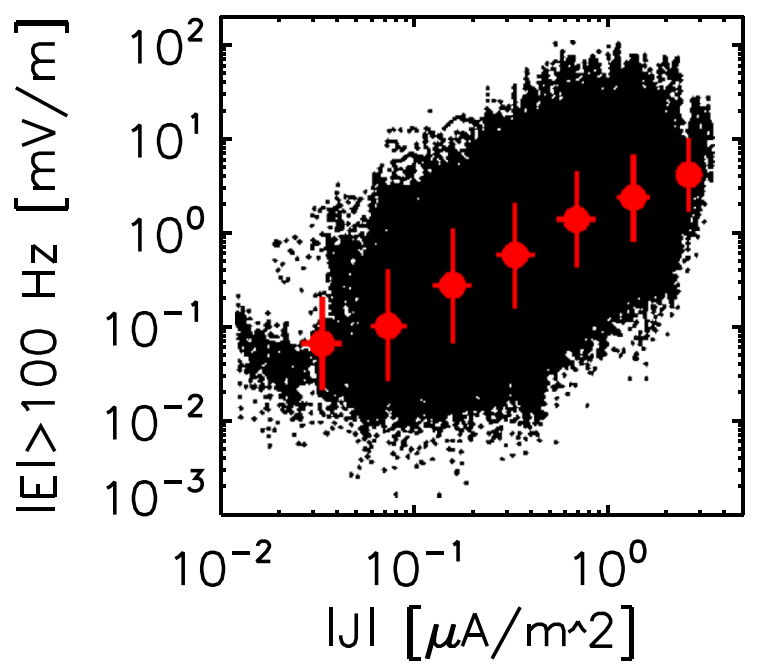

Figure 5. Comparison of current density magnitude from SC1 moments as a function of the magnitude of electric fields above $100 \mathrm{~Hz}$. The red dots indicate bin averages. generally shown not to be phase standing with the shock. Given that we make no such assumption, the currents reported here are viewed to be more accurately determined for the whistlers in the frequency range considered here.

The currents carried by the LF whistlers appear to be sufficiently large to excite electrostatic (ES) waves in the interval. Evidence of this is provided in Figure 4e, which gives normalized electric field PSDs. Localized bursts of ES waves are seen to occur quasiperiodically throughout the interval, as evidenced by the broadband enhancements that extend from $\sim 100 \mathrm{~Hz}$ to Nyquist. These have no counterpart in the normalized magnetic field PSDs (see Figure 4f). The waveform of the electric fields high-pass filtered above $100 \mathrm{~Hz}$ is shown in Figure $4 \mathrm{~g}$ and the magnitudes are given in Figure $4 \mathrm{~h}$. The ES waves are bursty in nature and have peak amplitudes generally peaking at a few to $50 \mathrm{mV} / \mathrm{m}$, with one instance (near 0805:43.7 UT) reaching nearly $100 \mathrm{mV} / \mathrm{m}$ (off scale). With some exceptions, the broadband ES wave signatures are collocated with gross current density enhancements in Figure $4 \mathrm{~b}$ throughout the interval. Fiducial vertical lines (cyan) delimit select adjacent intervals of current density maxima from $\mathrm{SC} 1$, as a visual aid. There are instances where there are some misalignments between peaks in ES waves and peaks in total currents. Much of these are related to the ES waves being preferentially peaked at parallel or perpendicular currents, which are not always exactly peaked with the total current (compare Figure $4 \mathrm{c}$ with Figure $4 \mathrm{~g}$ in middle and latter parts of the interval for associations with perpendicular and parallel currents, respectively.) This correspondence indicates that the localized regions of enhanced current are important sites for the generation of these waves.

To verify the correspondence between LF whistler waves and the ES waves, Figure 5 compares the current density magnitude from SC1 moments as a function of the magnitude of electric field above $100 \mathrm{~Hz}$ sampled in the interval spanning 0805:29.5 to 0805:54.9 UT, which covers the foot and part of the magnetic ramp in Figure $1 \mathrm{~d}$. The current densities were interpolated to the ES wave times for the comparison. The comparison reveals a positive correlation between the ES wave electric fields and currents, with a linear correlation coefficient in log space found to be $r=0.6$. The correlation is qualitatively consistent with the results of the study by Wilson et al. (2014b), who reported a similar correlation at shocks with lower Mach numbers than reported here. The broad spread in the data is related to the bursty nature of the ES waves in currents that are sampled at a lower cadence in addition to the slight misalignments with total currents mentioned above, owing to waveforms being more preferentially peaked at parallel or perpendicular currents, which are not always exactly peaked at total current maxima. Another contributing factor is there are likely other dependencies associated with the growth of these ES modes, such as electron and ion temperatures, that are at play here, which need further analysis that is outside the scope of this paper. These correlative results support the notion that the ES waves above $100 \mathrm{~Hz}$ are generated by current instabilities induced by the large amplitude whistler waves present in the layer.

With the currents and electric fields carried by these waves, the whistlers can possibly dissipate their energy into the plasma and vice versa. The dissipation rate as measured by $\mathbf{J} \cdot \mathbf{E}$ is a frame-dependent quantity, due to the frame dependence of the electric field. Here, we are interested in the energy transfer rates attributed to the LF whistlers in the shock NIF $\mathbf{J} \cdot \mathbf{E}_{\mathrm{NIF}}$. Thus, prior to computing, the electric field measured in the spacecraft frame was transformed to NIF using the expression $\mathbf{E}_{\mathrm{NIF}}=\mathbf{E}_{\mathrm{SC}}+\left(v_{\mathrm{sh}} \hat{\mathbf{n}}+\mathbf{v}^{\mathrm{NIF}}\right) \times \mathbf{B}$, where $\mathbf{v}^{\mathrm{NIF}} \equiv$ $\hat{\mathbf{n}} \times\left(\mathbf{V}_{\mathrm{SW}} \times \hat{\mathbf{n}}\right)$. It should be noted that the current used to compute $\mathbf{J} \cdot \mathbf{E}_{\mathrm{NIF}}$ is based on values determined from electron and ion moments from SC1 (i.e., $\mathbf{J} \equiv \mathbf{J}_{\mathrm{mom} 1}$ ).

Figure 6 shows dissipation rates in the foot and ramp of the shock from SC1. An overview of the background magnetic field (black curve) and the $1 \mathrm{~s}$ boxcar-averaged magnetic field (green curve) is shown in Figure 6a for context. The vertical lines indicate the region of interest in the foot and ramp where dissipation rates are determined. Figure $6 \mathrm{~b}$ displays the burst sampled magnetic field from FGM (black curve) and the $1 \mathrm{~s}$ boxcar averaged magnetic field (green curve) in the zoomed-in interval. The normalized power spectral density of the magnetic field (from merged FGM and SCM measurements) and polarization are given in Figures $6 \mathrm{c}$ and $6 \mathrm{~d}$, respectively. Values for $\mathbf{J} \cdot \mathbf{E}_{\mathrm{NIF}}$ for waves at frequencies $<30 \mathrm{~Hz}$ are given in Figure $6 \mathrm{e}$. This 


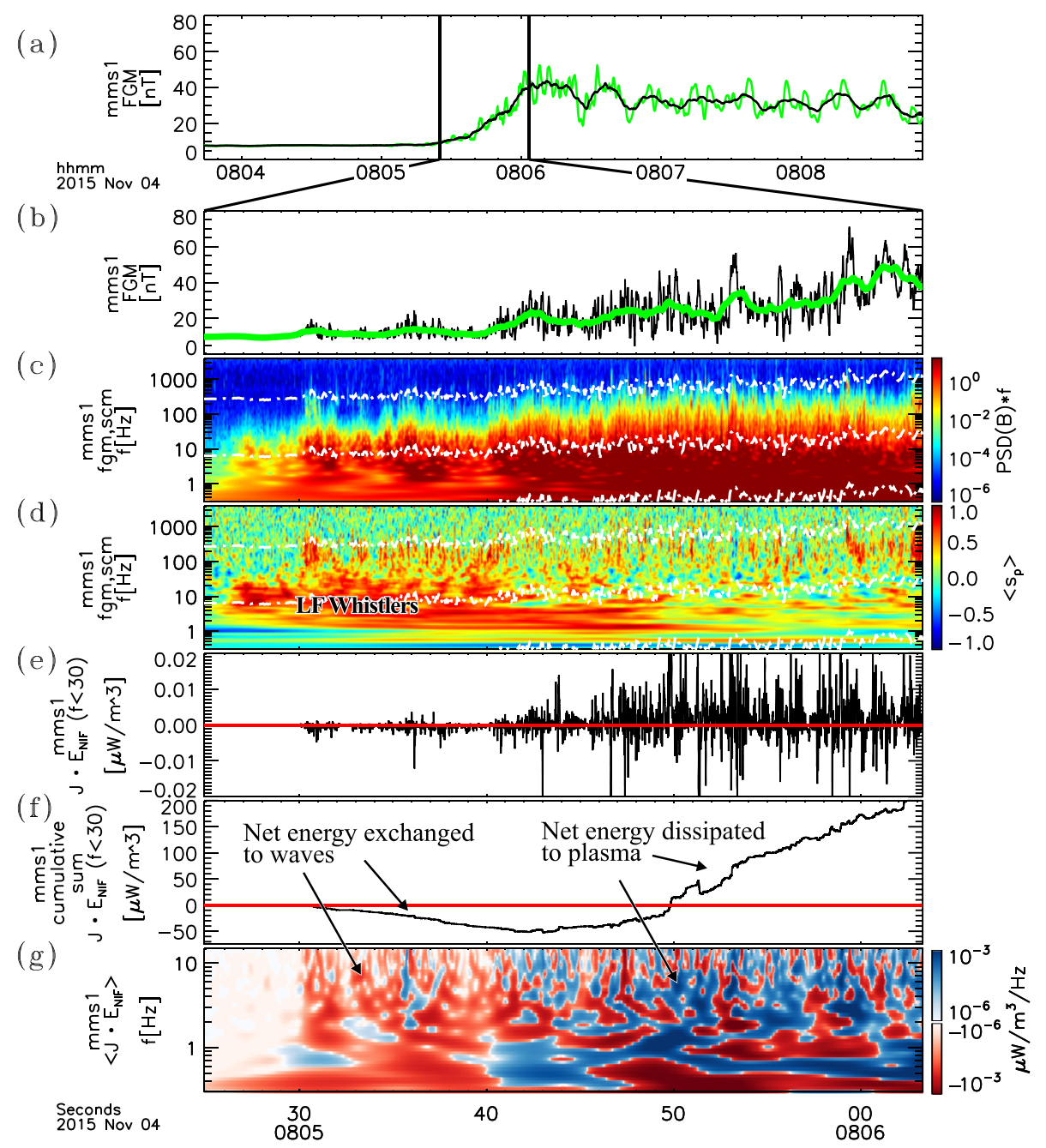

Figure 6. (a) An overview of the magnetic field magnitude from SC1. Also shown in zoomed-in view of foot and ramp region are the (b) the magnetic field magnitude, (c) normalized power spectra of magnetic field, (d) magnetic field polarization, (e) the dissipation rate in NIF $\mathbf{J} \cdot \mathbf{E}_{\mathrm{NIF}}$, (f) cumulative sum of $\mathbf{J} \cdot \mathbf{E}_{\mathrm{NIF}}$ for fields at $<30 \mathrm{~Hz}$, and (g) dynamic spectra of $\mathbf{J} \cdot \mathbf{E}_{\mathrm{NIF}}$.

frequency range encompasses most of the LF whistler range, as indicated by their right-hand polarization signatures in Figure 6d. Positive values for $\mathbf{J} \cdot \mathbf{E}_{\mathrm{NIF}}$ correspond to energy exchange from the electromagnetic fields to the plasma, while negative values represent energy being extracted from the plasma and deposited to the electromagnetic fields. In this interval the magnitude of the dissipation rate $\left|\mathbf{J} \cdot \mathbf{E}_{\mathrm{NIF}}\right|$ increases with increasing penetration in the layer, and has values ranging from a few thousandths to a few hundredths of $\mu \mathrm{W} / \mathrm{m}^{3}$, with only a few (off-scale) instances reaching up to $0.1 \mu \mathrm{W} / \mathrm{m}^{3}$. Values for $\mathbf{J} \cdot \mathbf{E}_{\mathrm{NIF}}$ are somewhat bursty, appearing to be concentrated with the substructure enhancements. Interestingly, the values for $\mathbf{J}$. $\mathbf{E}_{\mathrm{NIF}}$ are shown to be preferentially negative definite in the first half of the interval, but then transition to preferentially positive definite in the second half of the interval. To get a sense of the net amount of energy exchanged per unit volume per unit time, Figure 6 f gives the cumulative sum of $\mathbf{J} \cdot \mathbf{E}_{\mathrm{NIF}}$ from the LF whistlers. The trend shows that in the region, where the LF whistlers are dominantly right-hand polarized in the foot/first half of layer, there is a net negative sum, indicating that a net energy exchange from the plasma to the LF waves is occurring here. However, with further penetration into the ramp and just before the overshoot (second half of the interval), a net accumulated energy is observed to be dissipated into the plasma. In this latter region the wave polarizations become less discernible, with whistlers being less well-defined owing to what appears to be admixtures of modes in a given frequency bin, which disrupt their polarization within one or two cycles. To get a sense of relative contributions as a function frequency, Figure $6 \mathrm{~g}$ shows the dynamic spectra of the dissipation rate determined from $<\mathbf{J}(f) \cdot \mathbf{E}(f)>=\frac{1}{2} \operatorname{Re}\left\{\mathbf{J}(f) \cdot \mathbf{E}^{*}(f)\right\}$ (e.g., Swanson, 
1989). Here, the dot product is performed in frequency space with Morlet wavelet transformed components of the current $\mathbf{J}(f)$ and electric field complex conjugate $\mathbf{E}^{*}(f)$. The $<>$ represents an average over the wave period. In Figure $6 \mathrm{~g}$ red indicates energy exchanged from the plasma to the waves, while blue indicates wave energy being dissipated into the plasma. Though the range is somewhat limited, due to the electron sampling, it is clear that energy is being preferentially extracted from the plasma into the waves over the full frequency range in the first half of the interval. At about 0805:40 UT and after, localized patches of positive (blue) values of $\langle\mathbf{J}(f) \cdot \mathbf{E}(f)>$ are seen intermixed with negative (red) values throughout the rest of the interval, indicating that enhanced dissipation of electromagnetic wave energy into the plasma is occurring. The behavior in the foot, where a net energy exchanged from the plasma to the fields is observed, may be an indication of being in the LF whistler source region. It is worth noting that the kinetic energy in the ion beam is over 3 orders magnitude larger than the energy in the oblique whistlers, as evidenced in PIC simulations of the MTSI-FD (Muschietti \& Lembège, 2017). Thus, there is no measurable reduction of the beam drift expected in the interval. The subsequent behavior, where a net energy is exchanged into the plasma, indicates that this is the region in the shock layer where most of the irreversible plasma heating contributions that arise from the LF wave-particle interactions occurs. This trend suggests a scenario whereby the waves are generated in the foot and subsequently blown into the ramp, where their energy gets dissipated into plasma heating. Whether the observed energy conversion trend is characteristic of high Mach number shocks or not and to confirm the suggested (or some other) scenario will require careful analysis of more events under differing conditions coupled with detailed modeling, which is left for future work.

\subsection{Impacts on Electron Anisotropy}

Intense LF whistlers are also observed to impact electron distributions and hence temperature variations in the layer. To demonstrate this, Figure 7 shows magnetic field and electron data sampled in the first half of the ramp region. The magnetic field magnitude from FGM given in Figure 7a shows large amplitude variations (approaching $\delta B / B_{\circ} \sim 1$ ). These are due to a broad spectrum of right-hand polarized obliquely propagating LF whistlers at frequencies near and below a few tens of Hertz. To verify this, Figures $7 \mathrm{~b}-7 \mathrm{~d}$ show the normalized power spectra of the magnetic field sampled by SCM and associated polarization and propagation angle with respect to the background magnetic field vector determined from the Means method (Means, 1972). The intense LF whistlers are most apparent in the dynamic polarization spectra and correspond to the red signatures (indicative of right-hand polarization) spanning from $1 \mathrm{~Hz}$ to a few tens of $\mathrm{Hz}$ in Figure 7c. The oblique propagation angle is manifested by the yellow to green color in Figure 7d over this same frequency range.

To examine how the LF whistler induced field variations affect electrons, the electron temperatures parallel $\left(T_{e\|\|}\right)$ and perpendicular $\left(T_{e \perp}\right)$ to the magnetic field are exhibited as the black and red curves in Figure 7e. Also, the electron temperature anisotropy, defined as $\mathcal{A}_{e}=T_{e \perp} / T_{e \|}$, is given in Figure 7f. With some exceptions, the anisotropy varies quasiperiodically at few $\mathrm{Hz}$ cadence from preferentially perpendicular anisotropy $\left(\mathcal{A}_{e}>1\right)$ to preferentially parallel anisotropy $\left(\mathcal{A}_{e}<1\right)$ in the interval. Anisotropy enhancements tend to occur at localized maxima in the magnetic field. This correspondence is attributed to enhanced perpendicular temperatures, which are strongly correlated with the magnetic field (linear correlation coefficient $r=0.64$ ) and also generally (but not always) accompanied by depressed parallel temperatures in the region. As a visual guide, fiducial lines (purple dotted) are plotted at local minima in the electron temperature anisotropy having values $\lesssim 1$. Though bounding gross peaks in the anisotropy, the fiducial lines effectively bound maxima in the magnetic field indicating a causal connection. To examine electron features attributed to the anisotropy variations, Figures $7 \mathrm{~g}-7 \mathrm{i}$ depict energy flux pitch angle spectra of electrons at energies ranging from $10-50 \mathrm{eV}, 50-100 \mathrm{eV}$, and 100-500 eV, respectively. These energy ranges span the thermal and suprathermal electron populations in the interval. Note, with an inward pointing magnetic field, pitch angles $(\alpha)$ less than $90^{\circ}$ represent ingoing electrons of solar wind origin, while pitch angles larger than $90^{\circ}$ represent upstream directed/outgoing electrons. Close inspection indicates that anisotropy (and thus magnetic field) enhancements are associated with electron energy flux pitch angle spectra that are preferentially enhanced near $90^{\circ}$ and/or have prominent energy flux dropouts near $180^{\circ}$ at energies $\leq 100 \mathrm{eV}$ (blue to cyan in Figure $7 \mathrm{~g}$ and blue to green in Figure $7 \mathrm{~h}$ ). In contrast depressed anisotropy values are associated with preferential field-aligned (and/or antialigned) energy fluxes, which are often accompanied with somewhat depressed values in the vicinity of $90^{\circ}$ pitch angles. 


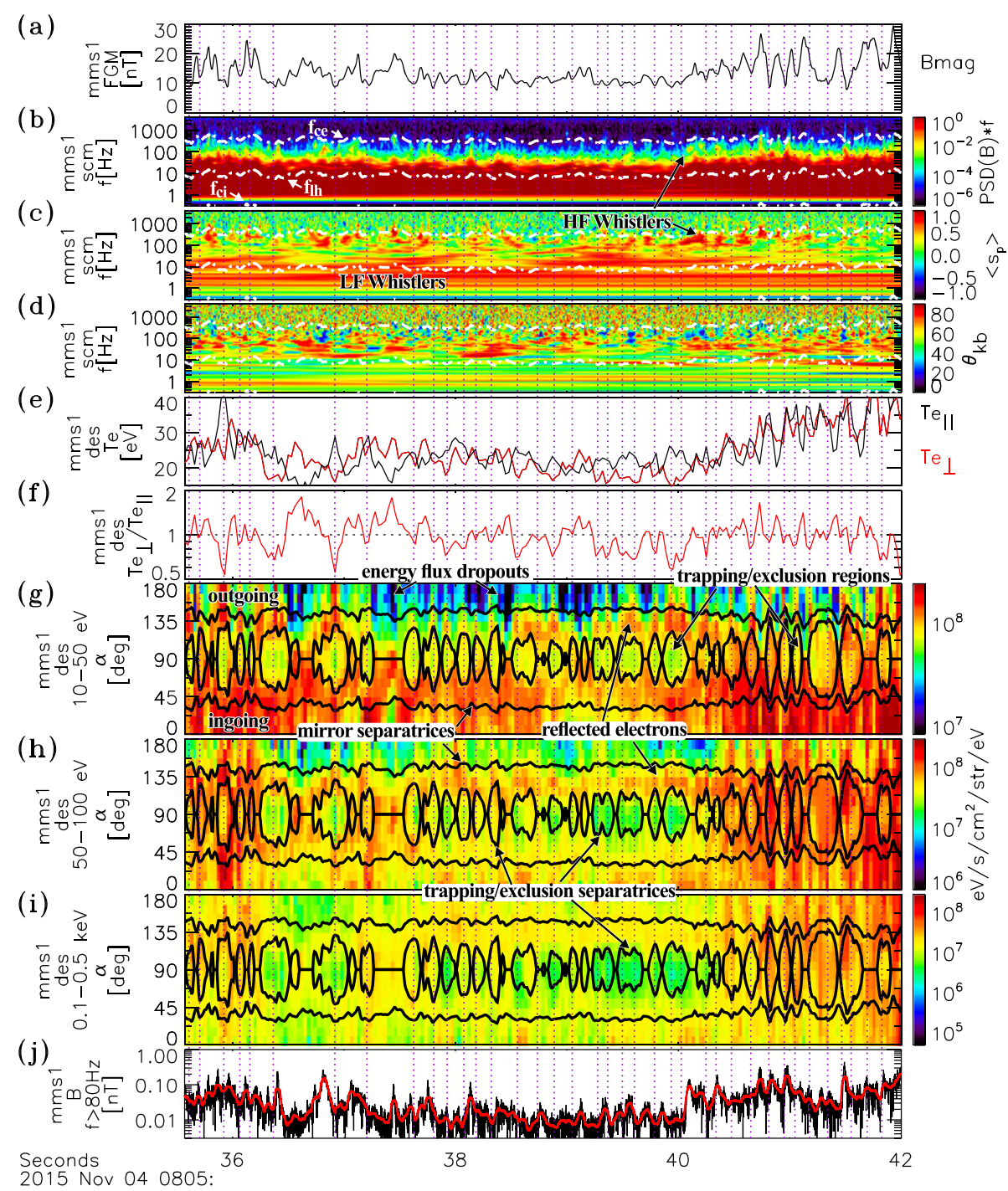

Figure 7. (a) Magnitude of magnetic field from FGM, (b) normalized PSD of magnetic field from SCM, (c) polarization, (d) wave-normal angle with respect to $\mathrm{B}$, (e) electron temperature perpendicular (red) and parallel (black) to the magnetic field, (f) electron temperature anisotropy. The energy flux pitch angle spectra for electrons at energies ranging from $(\mathrm{g}) 10-50 \mathrm{eV}$, (h) $50-100 \mathrm{eV}$, and (i) $100-500 \mathrm{eV}$, and magnetic field amplitude of waves (j) above $80 \mathrm{~Hz}$ (black curve), with the red curve indicating the average amplitude obtained using a $50 \mathrm{~ms}$ boxcar sliding window.

The observed electron features together with the observations of increasing (and also decreasing) $T_{e \perp}$ with increases (decreases) in the magnetic field may be explainable via the interaction of magnetized electrons in the intense, whistler induced, short-scale, nonmonotonic magnetic fields occurring in the layer in a manner qualitatively similar to that reported by Hull et al. (2001) in the context of nonmonotonic features of the shock macroscopic magnetic field profile. Electrons are considered magnetized when the gyroradius is much smaller than the spatial scale of the magnetic variations $r_{g} / L_{B} \ll 1$. Note that the typical thermal electron gyroradius in the interval is $r_{g} \equiv V_{\text {te }} / \omega_{\text {ce }} \sim 1 \mathrm{~km}$, while a $500 \mathrm{eV}$ electron is $r_{g}^{500} \sim 4.4 \mathrm{~km}$. Verifying the magnetized behavior, these values are much smaller than the shortest whistler wavelength $\left(\lambda \approx \lambda_{i} / 3\right)$ documented in Table 1, which yield gyroradius to wave-scale ratios on the order of 0.04 and 0.15 for thermal and $500 \mathrm{eV}$ electrons, respectively. Such a model, through magnetic moment conservation $\left(\mathcal{E}_{\perp} / B=\right.$ const, where $\mathcal{E}_{\perp}$ is the electron-perpendicular energy), predicts enhancements (decreases) in the perpendicular energy of electrons in increasing (decreasing) magnetic fields. By energy conservation $\mathcal{E}_{\text {tot }}=\mathcal{E}_{\perp}+\mathcal{E}_{\|}$, the parallel energy is expected to decrease (increase) with increases (decreases) in the perpendicular energy of 
the electrons in the absence of an electrostatic electric field, which can energize the electrons. This expectation is qualitatively similar to the variations in the perpendicular and parallel temperatures observed in the interval.

To better assess how the nonmonotonic magnetic fields associated with the whistlers are affecting the electron distribution features, we can use magnetic moment and energy constraints in this approximation to determine the boundaries that separate different classes of electron trajectories. It is important to emphasize that the potential generally cannot be neglected for sufficiently low energy electrons. However, as will be demonstrated shortly, this approximation does a very good job in assessing the imprints of the nonmonotonic magnetic fields on the electrons at the energies considered. This is because the potential the electrons see is small over much of the foot region under consideration. The basic approach is to identify different classes of electron orbits that have access to different parts of the shock. In a magnetic profile with multiple magnetic minimaxes electrons with sufficient parallel kinetic energy will make it through the multiple magnetic bottles to a given location, while those that do not will get reflected. Also, depending on pitch angle, nonmonotonic fields can trap magnetized electrons or prevent magnetized electron orbits from reaching locations between field enhancements (i.e., within local minima) (Hull et al., 2001). The former situation can occur in the region between a pair of growing magnetic field enhancements, for example, while the latter can occur between a preexisting pair of magnetic maxima. Thus, based on these considerations and using these constraints, one can determine two types of separatrices: (1) the separatrix (denoted the mirror separatrix) that delineates electron orbits that can connect to both sides of the shock (free streaming orbits) and those that get reflected, and (2) the separatrix for trapped (or excluded) electron orbits. The former depends on the maximum macroscopic magnetic field between the upstream and downstream sides of the shock, while the latter depends on local magnetic field maxima. A detailed discussion for how these boundaries were determined is given in Appendix B1.

The mirror separatrix boundaries are indicated by the solid curves near $30^{\circ}$ and $150^{\circ}$ pitch angles in Figures $7 g-7 i$. Separatrix boundaries delineating the trapping/exclusion regions determined via magnetic moment and energy considerations are indicated by the closed boundaries about $90^{\circ}$ pitch angles in Figures $7 g-7 i$. It can be seen in Figures $7 g-7 i$ that the two sets of separatrices (denoted mirror and trapping/exclusion) well organize the observed features in the electron pitch angle spectra suggesting that these features are strongly controlled by the interaction of magnetized electrons in the nonmonotonic magnetic fields. Particularly, the close correspondence between variations in the trapping/exclusion boundaries and the edges of the depressed electron fluxes indicates that these depressed regions are indeed the result of magnetic mirroring that prevents magnetized electrons that have pitch angles near $90^{\circ}$ from gaining access to these local magnetic minima regions. These regions are not empty but appear as remnants of voids that may be partially filled by pitch angle scattering of electrons via waves into these regions of phase space. Also, electrons are seen to occur at pitch angles $90^{\circ}<\alpha \lesssim 150^{\circ}$ between the trapping boundary and the outer mirror separatrix boundary. These are the mirrored counterpart of electrons at pitch angles $30^{\circ} \lesssim \alpha<90^{\circ}$. Finally, the energy flux dropouts/depressions near $180^{\circ}$ seen in Figure $7 \mathrm{~g}$ (and to a lesser extent Figure $7 \mathrm{~h}$ ) indicate a dearth of electrons that leak to these regions of the shock from the magnetosheath. Though not considered in the simplified discussion above, these are interpreted as the result of a shock potential that acts to prevent low-energy magnetosheath electrons from escaping to the upstream side leaving only the tail of the energy flux spectra corresponding to "postshocked" electrons that do have access to this region from the downstream side. Such depressed loss cone features have been seen upstream (Feldman et al., 1983; Fitzenreiter et al., 1990) and in the foot of Earth's bow shock (e.g., Hull et al., 2001; Scudder, Mangeney, Lacombe, Harvey, Aggson, et al., 1986), but not with the unprecedented resolution provided by the MMS mission. The potential is more important for lower-energy electrons (e.g., Figure $7 \mathrm{~g}$ ) and acts to reduce the effects of mirroring by opening up the source/loss cone throat somewhat so as to maintain normal current continuity (Hull et al., 2001; Scudder, 1995). A rough estimate of the deHoffmann-Teller potential (which is most relevant for electrons) from the electron energy equation (Hull et al., 2000) is $\Phi^{\mathrm{HT}} \sim 15 \pm 10 \mathrm{~V}$ for the middle part of the interval but increases to $40 \pm 15 \mathrm{~V}$ in the latter part (after $\sim 0805: 41$ ). The effect of this is to introduce energy-dependent corrections that narrow the mirror separatrix somewhat closer to $90^{\circ}$ at lower electron energies. The trapping/exclusion separatrices are much less affected, since what matters is the local change of the potential the electrons see at and between local field minima, which is small $\left(\delta \Phi^{\mathrm{HT}} \lesssim\right.$ $10 \mathrm{~V}$ ) for most of the region, except at the very beginning and latter part (after $\sim 0805: 41$ ), where the changes 
approach 20 to $30 \mathrm{~V}$ in a couple of instances. Nevertheless, the comparisons do indeed show that the separatrices, which neglect the potential corrections, do a very good job of elucidating the topology of the electron pitch angle spectra over the energy ranges considered in response to magnetic field variations in the interval. While it is clear that these results indicate that the whistlers are affecting the electron distribution and hence temperatures in the region, a detailed characterization of electron features in response to variations in the fields, including the effects of the potential, requires further analysis that is beyond the scope of this current paper and is left for future work.

The anisotropies induced in the electron distributions are sufficiently strong to excite short spatial scale high-frequency whistlers. This is evidenced by the near coincidental occurrence of short duration, small amplitude, magnetic field enhancements above $80 \mathrm{~Hz}$ shown in Figure $7 \mathrm{j}$ with peaks in $\mathcal{A}_{e}$ or loss cone features in the electron pitch angle spectra shown in Figures $7 \mathrm{f}$ and $7 \mathrm{~g}$, respectively. These enhancements are attributed to relatively narrow-band, quasi-periodic right-hand polarized signatures near and above $100 \mathrm{~Hz}$ in Figure 7c. These whistlers typically propagate quasi-parallel (or anti-parallel) with respect to the background magnetic field (i.e., $\theta_{k b} \lesssim 30^{\circ}$ indicated by cyan to blue colors in Figure $7 \mathrm{~d}$ ). This correlation indicates that the anisotropies/loss cone features in the electron distribution induced by the LF whistler are a source of free energy for the generation of these waves. The anisotropies from moments, with peak values typically reaching $1.3 \pm 0.2$ with a few instances reaching up to 1.8 in the interval, are similar to the values reported in previous studies based on a more coarser data set (Hull et al., 2012). With the local electron beta $\beta_{e}$ being $\sim 0.5$, the anisotropies yield relatively slow values for the maximum growth rate implied from linear theory. Namely, $\gamma \sim 1-10 \omega_{\mathrm{ci}}$, where $\omega_{\mathrm{ci}}$ is the ion cyclotron frequency (e.g., Gary, 1993). From the characteristics of the HF whistlers one can compute the energy of the resonant electrons. Depending upon the resonance considered (Cerenkov $n=0$, cyclotrons $n=1$, or -1 ) the energy ranges from 15 to $50 \mathrm{eV}$. So, it is the pitch angles of the low-energy electrons from Figure $7 \mathrm{~g}$ that drives the waves unstable, even though the temperature ratio of the whole distribution function is not large. Indeed close inspection of electron distributions does reveal the presence of large anisotropies (e.g., $f_{\perp} / f_{\|} \sim 3-6$ ) associated with loss cone features (related to the energy flux dropouts) that are coincident with the HF whistlers. Such large anisotropies are expected to enhance growth rates enabling the HF whistlers to grow to sufficient amplitudes in the localized regions where they are observed (e.g., Veltri \& Zimbardo, 1993). An assessment of the effects of large loss cone-associated anisotropies in the observed electron distributions on driving such whistlers requires a more detailed analysis that is beyond the scope of this current paper and thus is left for future work.

\subsection{Assessment of Source Mechanisms for LH Whistlers}

Accurate determinations of the wave properties (e.g., wave vectors, wave lengths, and phase velocities) in combination with high-time-resolution plasma data enables an assessment of source mechanisms for the generation of the intense whistler waves that are observed near the lower-hybrid frequency. Given that the waves were shown in section 3.2 to be propagating upstream at oblique angles with respect to the magnetic field and have wavelengths that are at or just below the ion inertial length scale, we can eliminate the MTSI-SD as a source mechanism. Recall that, in order to be in resonance with the incoming solar wind ions, whistler waves excited by this mechanism are expected to have wave vectors preferentially oriented in the downstream direction nearly perpendicular to the background magnetic field. The wave vector propagation direction does favor two possible mechanisms: (1) MTSI-FD and (2) dispersive radiation of whistler waves upstream. Discriminating between these two sources can be challenging, since both mechanisms can give rise to whistlers with upstream directed phase speeds and also Poynting fluxes. In both cases the whistler waves need not be phase standing with the shock in the shock frame. A possible way to discriminate between the two candidates is to examine whether or not there is a relationship between the wave propagation vector and the reflected ion beam properties. The dispersive radiation model is basically a fluid model and ignores entirely the reflected ion beam. Thus, we do not expect there to be any connection with the reflected ion beam properties. However, the MTSI-FD source mechanism involves the reflected ions, which implies a measurable connection between the waves and the reflected ion beams, if we are in the source regions of these waves.

One condition that should be met for the MTSI-FD mechanism is that the phase speed in the plasma frame $V_{p}$ should satisfy the Cerenkov resonance condition with the beam. If $\theta_{k v}$ is the angle between the wave vector direction $\hat{\mathbf{k}}$ and the reflected ion beam velocity direction $\hat{\mathbf{V}}_{b}$, the Cerenkov resonant velocity along 


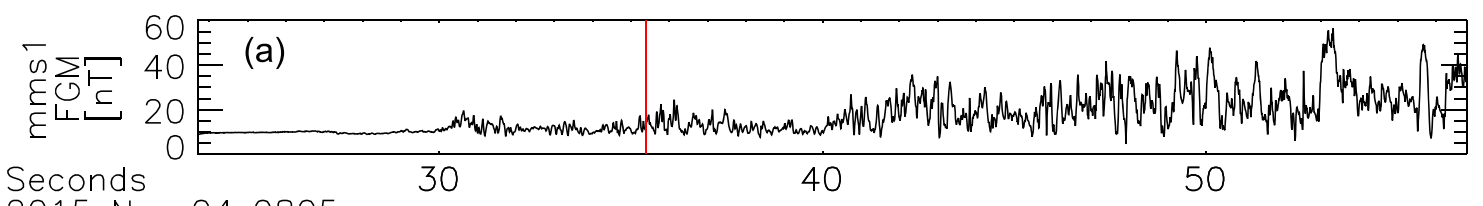
2015 Nov 04 0805:

(b) 08:05:35.194-08:05:35.344
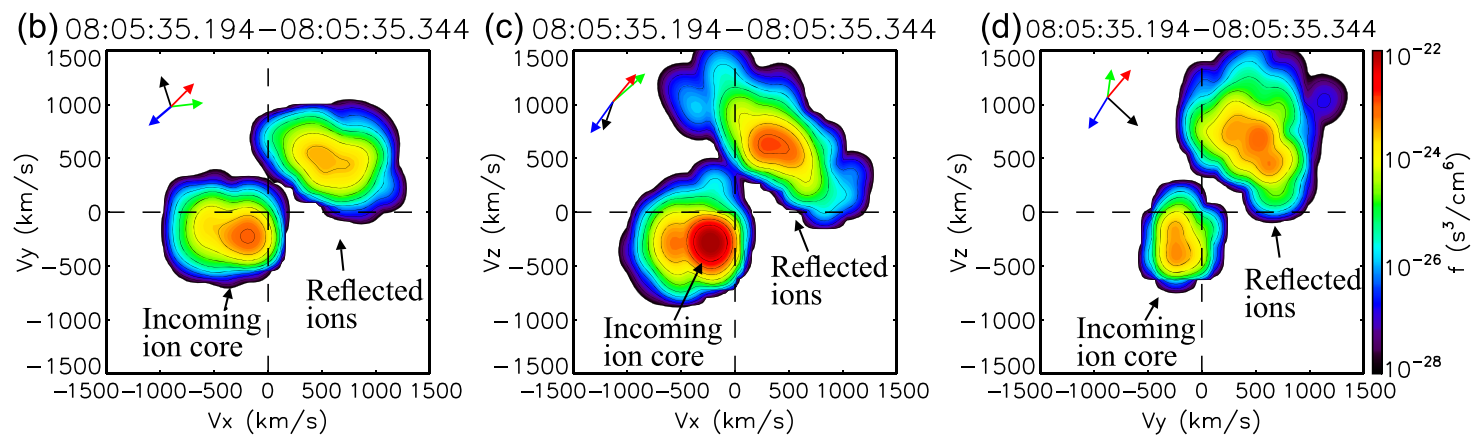

Figure 8. (a) Magnetic field magnitude from FGM, and ion distribution in the plasma rest frame sampled at 0805:35.293 UT projected onto the GSE (b) $V x$ - $V y$, (c) $V x-V z$, and (d) $V x$-Vy velocity space planes, respectively. Also indicated in the upper left-hand corner of panels (b)-(d) are the projections of the wave normal (green arrow), magnetic field (black arrow), reflected ion beam velocity (red arrow) and the solar wind ion velocity (blue arrow) unit vectors.

the beam direction is given by

$$
V_{r}=V_{p} / \cos \theta_{k v}
$$

Unlike the solar wind ion core, the reflected ion beam is broad with a velocity spread that can be on the order of $100 \mathrm{~km} / \mathrm{s}$ or more. To account for the beam broadness, one can write the resonance condition as

$$
V_{r}=\kappa V_{b} \text {. }
$$

For the MTSI-FD model one expects the Cerenkov resonance to be on the positive slope created by the beam, that is, the factor $\kappa$ lies in a range $1-\Delta V_{b} / V_{b} \lesssim \kappa<1$. In contrast, for the dispersive radiation model one expects the factor $\kappa$ to take any value, including $\kappa<0$ or $\kappa>1$, since there should be no causal link to the ion beam. One can just expect a selection effect based on the ability of the whistlers to stand or propagate upstream and be seen at a given location in the shock, which favors large values of $V_{r}$.

In order to test the criteria we need to determine the reflected ion beam velocity $\mathbf{V}_{b}$ in the rest frame. As an example of the features typically observed in the layer, an ion distribution in the rest frame projected onto the GSE $x-y, x-z$, and $y-z$ planes are shown in Figures $8 \mathrm{~b}-8 \mathrm{~d}$. This sample corresponds to the $10 \mathrm{~Hz}$ waveform example event observed at 0805:35.293 UT previously described in section 3.2. Note that the GSE coordinate system is incidentally close (within a few degrees) to the NIF coordinate system, with the outward pointing shock normal $\hat{\mathbf{n}}_{\mathrm{RH}}=[0.9867,0.1615,-0.01916]$ being aligned primarily along GSE- $x$, the out-of coplanarity component $\hat{\mathbf{s}}=[-0.0267,0.2773,0.9604]$ is nearly along GSE- $z$, and the third component $\hat{\mathbf{t}}=[0.1604,-0.9471,0.2779]$ completes the orthonormal basis and is nearly antiparallel to GSE- $y$. As shown in Figures $8 \mathrm{~b}-8 \mathrm{~d}$, the ion distribution function is primarily composed of two components. There is a denser cooler component corresponding to the incoming core solar wind ions with a velocity component $V_{x}<0$. By contrast, the reflected beam is the much broader signature with a velocity component in the positive $x$ direction, $V_{x}>0$. To estimate $\mathbf{V}_{b}$, we bracketed the reflected beam in 3-D velocity space and obtained the peak. In this example $\mathbf{V}_{b}$ is estimated at $792[0.537,0.542,0.646] \mathrm{km} / \mathrm{s}$, which is nearly perpendicular to the ambient magnetic field with $\theta_{\mathrm{vb}}=98^{\circ}$ and preferentially oriented toward the wave vector with $\theta_{\mathrm{kv}}=32^{\circ}$.

The direction of the beam $\hat{\mathbf{V}}_{b}$ introduces a second anisotropy axis besides that of the ambient, local magnetic field $\hat{\mathbf{B}}_{o}$. We have determined the reflected ion beam velocities for the events listed in Table 1 and noticed that the wave vectors appear to lie close to the plane $\Psi$ containing $\hat{\mathbf{V}}_{b}$ and $\hat{\mathbf{B}}_{o}$. Each of these events occurred in the presence of a well-defined reflected ion beam, which improves the accuracy of the comparison. The results are listed in Table 2, where values are expressed in the plasma frame and GSE coordinates used for vectorial quantities. The table includes the beam velocity magnitude $V_{b}$ and direction $\hat{\mathbf{V}}_{b}$, the ratio $V_{b} / V_{A}$, the angle $\theta_{\mathrm{vb}}$ between $\hat{\mathbf{V}}_{b}$ and the background magnetic field, the angle $\theta_{\mathrm{kv}}$ between $\hat{\mathbf{k}}$ and $\hat{\mathbf{V}}_{b}$, the resonance 
Table 2

Reflected Ion Beam and Resonance Diagnostics

\begin{tabular}{lcccccccr}
\hline Time & $V_{b}(\mathrm{~km} / \mathrm{s})$ & $\hat{\mathbf{V}}_{\mathbf{b}}$ & $V_{b} / V_{A}$ & $\theta_{\mathrm{vb}}$ & $\theta_{\mathrm{kv}}$ & $V_{r}(\mathrm{~km} / \mathrm{s})$ & $V_{r} / V_{b}$ & $\theta_{k \Psi}$ \\
\hline $0805: 30.930$ & 823 & {$[0.71,0.22,0.61]$} & 8.3 & $104^{\circ}$ & $14^{\circ}$ & 642 & 0.78 & $-6^{\circ}$ \\
$0805: 31.533$ & 869 & {$[0.64,0.27,0.72]$} & 9.1 & $97^{\circ}$ & $31^{\circ}$ & 599 & 0.69 & $-19^{\circ}$ \\
$0805: 31.580$ & 869 & {$[0.64,0.27,0.72]$} & 9.6 & $97^{\circ}$ & $25^{\circ}$ & 608 & 0.70 & $-2^{\circ}$ \\
$0805: 33.338$ & 778 & {$[0.76,0.32,0.56]$} & 8.9 & $103^{\circ}$ & $25^{\circ}$ & 631 & 0.81 & $-15^{\circ}$ \\
$0805: 33.338$ & 778 & {$[0.76,0.32,0.56]$} & 8.6 & $103^{\circ}$ & $25^{\circ}$ & 684 & 0.88 & $-4^{\circ}$ \\
$0805: 33.338$ & 778 & {$[0.76,0.32,0.56]$} & 8.9 & $103^{\circ}$ & $22^{\circ}$ & 723 & 0.93 & $+2^{\circ}$ \\
$0805: 34.646$ & 743 & {$[0.50,0.37,0.78]$} & 8.3 & $100^{\circ}$ & $16^{\circ}$ & 669 & 0.90 & $-0.2^{\circ}$ \\
$0805: 35.293$ & 792 & {$[0.54,0.54,0.65]$} & 8.5 & $98^{\circ}$ & $32^{\circ}$ & 774 & 0.98 & $-14^{\circ}$ \\
$0805: 35.397$ & 831 & {$[0.65,0.46,0.61]$} & 8.8 & $103^{\circ}$ & $19^{\circ}$ & 657 & 0.79 & $+9^{\circ}$ \\
$0805: 35.906$ & 740 & {$[0.44,0.66,0.61]$} & 7.6 & $98^{\circ}$ & $30^{\circ}$ & 681 & 0.92 & $-0.3^{\circ}$ \\
$0805: 37.832$ & 848 & {$[0.24,0.53,0.81]$} & 9.0 & $107^{\circ}$ & $29^{\circ}$ & 738 & 0.87 & $-19^{\circ}$ \\
$0805: 39.167$ & 868 & {$[0.48,0.13,0.87]$} & 9.4 & $114^{\circ}$ & $23^{\circ}$ & 773 & 0.89 & $-19^{\circ}$ \\
$0805: 41.091$ & 787 & {$[0.84,0.29,0.45]$} & 7.5 & $93^{\circ}$ & $40^{\circ}$ & 600 & 0.76 & $+14^{\circ}$ \\
$0805: 41.816$ & 520 & {$[0.67,0.40,0.62]$} & 4.3 & $85^{\circ}$ & $13^{\circ}$ & 475 & 0.91 & $+10^{\circ}$ \\
$0805: 41.902$ & 520 & {$[0.67,0.40,0.62]$} & 4.4 & $85^{\circ}$ & $28^{\circ}$ & 370 & 0.71 & $+7^{\circ}$ \\
$0805: 43.205$ & 497 & {$[0.58,0.42,0.70]$} & 4.7 & $94^{\circ}$ & $19^{\circ}$ & 428 & 0.86 & $-4^{\circ}$ \\
$0805: 47.919$ & 568 & {$[0.92,0.34,0.20]$} & 4.7 & $86^{\circ}$ & $42^{\circ}$ & 465 & 0.82 & $-8^{\circ}$ \\
\hline
\end{tabular}

velocity $V_{r}$, the factor $\kappa=V_{r} / V_{b}$, and the angle $\theta_{k \psi}$ between $\hat{\mathbf{k}}$ and the plane $\Psi$. As shown in Table 2, it is clear that the reflected ion beam velocities have varied amplitudes and orientations that rotate depending on location with respect to the specular reflection point. The results show that the wave vectors are preferentially oriented toward the beam with $\theta_{\mathrm{kv}}<45^{\circ}$ indicating their orientations are connected to the rotating ion beam. The angles $\theta_{k \psi}$ between the measured wave vectors and the plane $\Psi$ are also small (within $\pm 20^{\circ}$ ). Finally, the Cerenkov resonance velocity of the waves are less than, but reasonably close to the beam speed, with $0.7 \leq \kappa<1$ for the events listed. These favorable comparisons in combination with their consistent oblique upstream propagation with respect to the shock normal and magnetic field are consistent with the notion that these whistlers are causally linked to the reflected ion beam and that the source of these waves is the MTSI-FD mechanism.

Note that so far the analysis presented in this section does not make use of the linear dispersion relation. Phase speeds and frequencies are directly measured. The instability due to the reflected ions (MTSI-FD) was recently studied in an analysis combining self-consistently linear dispersion and PIC simulations (Muschietti \& Lembège, 2017). The wavelength characteristics were found to range from $\lambda=0.25 \lambda_{i}$ to $\lambda=0.9 \lambda_{i}$ for a propagation angle of $\theta_{\mathrm{kb}}=55^{\circ}$, with wave numbers in the range $7<k c / \omega_{\mathrm{pi}}<25$ (see Figures 9 and 12 in the above reference). Such values compare well with the measurements listed in Table 1, even though these measurements do not assume the linear dispersion relation. On the other hand, more work is needed to support the MTSI-FD mechanism. Indeed, the unstable region has a finite extent that consists of the shock's foot. Moreover, as the whistlers propagate they may be convected back toward the shock's ramp by the plasma flow. The observed trend in the energy conversion attributed to these whistler in the foot and ramp region may be an indication of this effect. To address this aspect and show that the waves can be amplified requires a careful assessment of their group speeds. The latter could act as a filter, which selects the unstable waves that are able to quasi-group stand in the flow. The question is quite involved and will be addressed in a future paper.

In the case of dispersive radiation a whole hierarchy of whistler waves are expected to be emitted, including the shortest scale whistlers at the resonance cone limit $\left(\cos \theta_{k b}=f / f_{\text {ce }}\right)$, as the nonlinear steepening of the ramp reaches the electron scale. This is expected to occur when the Fast mode Mach number is at or supersedes the nonlinear whistler critical Mach number (in cold plasma limit): $M_{F} \gtrsim M_{\mathrm{WNL}}=$ $\left|\cos \theta_{\mathrm{Bn}}\right|\left(m_{i} / m_{e}\right)^{1 / 2} / 2^{1 / 2}$. In this event $M_{F} \sim 6.7>M_{\mathrm{WNL}} \sim 4.2$, indicating that the shock is in the regime where reformation is expected to occur. For context, the whistler critical Mach number is estimated at $M_{W}=\left|\cos \theta_{\mathrm{Bn}}\right|\left(m_{i} / m_{e}\right)^{1 / 2} / 2=3$. As seen in Figure 1, a hierarchy of whistler waves is observed within the 
layer, including at high-frequency approaching a significant fraction of the electron cyclotron frequency. The bursts of whistlers near the lower-hybrid frequency being collocated with ramp substructure is an expected signature of the intrinsic time development of a reforming shock. However, in this model the whistlers are expected to have wave vectors that are preferentially oriented along the shock normal (e.g., Balikhin, de Wit et al., 1997; Dimmock et al., 2019). While some of the examples listed in Table 1 are inclined toward the normal, the majority of the LF whistlers reported here are propagating upstream at oblique angles with respect to the shock normal contrary to the expectations of dispersive radiation theory. It is also unclear whether the observed trend regarding energy conversion related to these waves in foot and ramp region can be explained by the model. One may expect that the intrinsic time development of bursts of whistlers in the ramp that are emitted upstream where their energy is dissipated would seem to result in a behavior opposite to the trend observed. These results along with the additional connection established between the wave vectors and the ion beam seem to favor the MTSI-FD over the dispersive radiation model. For the case of the HF whistlers, these have wavelengths approaching $\lambda \sim 10 \lambda_{e}$ (Hull et al., 2012). Such HF whistlers are not propagating oblique to the magnetic field but are generally observed with wave vectors that are quasi-parallel, as shown here and other studies (e.g., Hull et al., 2012; Oka et al., 2017; Zhang et al., 1999). With the magnetic field being nearly transverse to the shock surface, the wave vectors of these HF waves are oriented quasi-perpendicular to the shock normal, which is contrary to the predictions of the dispersive radiation model. Moreover, these HF whistlers do not appear to be the result of a transfer of energy to shorter scale related to a steepening front, but, as shown in section 3.4, appear to be locally generated by a microinstability driven by anisotropies in the electron distribution induced by the lower frequency whistler waves. While our results suggest that whistlers at lower hybrid and higher frequencies are driven by microinstabilities, this does not preclude dispersive radiation for whistler precursor substructure, which are present in the layer. Such substructure was recently estimated to have a width of $2-3 \lambda_{e}$ from multipoint Cluster observations (Dimmock et al., 2019).

\section{Summary and Conclusions}

The MMS mission is ideally suited to study the properties of whistler mode waves within Earth's bow shock. Namely, it provides for the first time the requisite high-time-resolution plasma and three-axis electric and magnetic waveform data simultaneously sampled in long-duration bursts on four spacecraft. These data allowed us to accurately quantify the wave vectors, phase speeds, and other properties within the foot and ramp region of Earth's bow shock. The accompanying plasma data, being sufficiently sampled over the waveform scales, enabled us to assess, at a high Mach number shock crossing, the source mechanism of intense LH whistlers and study how these waves interact with the plasma. Particularly advantageous was the ability to compute the currents these waves carry via multipoint measurements and also from particle drifts at subion inertial length scales for the first time. Moreover, the unique multipoint measurements enable an assessment of the spatial and temporal properties of these waves, and hence their impact on shock structure and stability. Key results documented in the paper are as follows:

1. Using the multipoint data, we showed unambiguously that whistlers near and just below the lower-hybrid frequency are composed of right-hand polarized wave packets that propagate upstream at oblique angles with respect to the magnetic field and shock normal. These LF whistlers have phase speeds ranging from a few hundred to nearly $1,000 \mathrm{~km} / \mathrm{s}$ in the shock frame (i.e., not phase standing). The waves have wavelengths in the range from $\sim 30$ up to $\sim 80 \mathrm{~km}\left(\sim 0.3\right.$ to $\left.1.3 \lambda_{i}\right)$. Through correlation analysis of multipoint measurements, we demonstrated that the waveforms are often quasi-stationary over the transit times of the spacecraft fleet with 11 to $18 \mathrm{~km}$ separations.

2. These LF whistlers were shown to be concentrated in large-amplitude precursor substructures that occur in the pedestal and into the shock ramp, indicating these are important sites for their generation. The observed properties of the waves (e.g., rest frame frequencies, wavelength, upstream directed wave vectors) along with the observation that the wave vectors are preferentially oriented toward the reflected ion beam in such a way as to satisfy the Cerenkov resonance condition are consistent with the modified two-stream instability source mechanism (a.k.a. kinetic cross-field streaming instability) excited by the relative drift between reflected ions and electrons (i.e., MTSI-FD). Though these are promising observational results, more work is needed to show that this mechanism has enough time to amplify the waves to amplitudes observed before being convectively blown back toward the ramp, which has inhomogeneities due to ramp substructure. The MTSI-SD was ruled out based on the observed fact that the 
typical wave vectors are propagating upstream and have wavelengths that are at or just below the ion inertial length scale, thus much longer than the electron inertial length expected for this instability. Aspects of the observed features are also consistent with expectations from the dispersive radiation mechanism including the wavelengths, frequencies, hierarchy of waves with upstream directed wave vectors. However, the oblique wave vector orientations with respect to the normal and connectivity with the reflected ion beam demonstrated in the paper are not in accordance with the predictions of the model, suggesting this might not be the mechanism.

3. The LF whistlers have intense magnetic fields $\left(\delta B / B_{\circ} \sim 0.1-1.0\right)$ and carry sizable currents (with $\left|J / J_{\text {vte }}\right|$ reaching 0.1 to 0.5 ) with characteristic spatial scales reaching at or below ion inertial length scales. While time-varying currents were observed, current variations were often found to be quasi-stationary over the short spacecraft separations and thus local crossing times, enabling accurate characterization of their properties. The currents are due to inhomogeneities in the whistler waveforms observed among the spacecraft. The enhancements in the whistler currents were observed to be collocated with moderately intense electrostatic waves, indicating that these currents were sufficiently large to excite these waves. These findings demonstrate that the LF whistler waves are affecting the internal structure of the shock in two ways: (1) by making significant, if not dominant, modifications to the shock fields and currents on subion inertial length scales and (2) by inducing large enough drifts for smaller-scale ES waves to be generated in a regime thought not possible in past studies.

4. Quantified energy exchange rates estimated from $\mathbf{J} \cdot \mathbf{E}_{\mathrm{NIF}}$ due to whistlers at $30 \mathrm{~Hz}$ and below are observed to range from a few thousandths to few hundredths of $\mu \mathrm{W} / \mathrm{m}^{3}$ in the foot and ramp region of the shock. Values for $\mathbf{J} \cdot \mathbf{E}_{\mathrm{NIF}}$ were shown to be preferentially negative in the foot, but transition to preferentially positive in the ramp region. These values were shown to yield net energy exchanged from the plasma to whistlers in the foot, whereas the waves are dissipated into the plasma in the ramp. The net energy conversion from the plasma to the waves in the foot may be an indication of being in the LF whistler source region. The much larger net energy exchange from the LF waves to plasma observed with further penetration into the ramp signifies that waves are making important contributions to irreversible plasma heating in this region. This trend suggests a scenario whereby the waves are generated in the foot and subsequently get blown into the ramp, where their energy accumulates due to the slowing flow and is dissipated into plasma heating. It is unclear whether the observed trend can be explained by the dispersive radiation model, since it would seem that the opposite behavior should be happening.

5. We showed that the LF whistler waves are impacting electron dynamics in the layer by inducing anisotropy variations in the electron distributions (and thus the perpendicular and parallel temperatures). For example, at magnetic field maxima, electron energy flux pitch angle spectra were preferentially enhanced near $90^{\circ}$ and/or had prominent energy flux dropouts near $180^{\circ}$ at energies $\leq 100 \mathrm{eV}$, while at magnetic minima the electrons tended to have a preferential field-aligned sense. Depending on location, electron energy flux depletions/depressions and, at times, trapped electrons were also observed in the vicinity of $90^{\circ}$ pitch angles in magnetic minima. We demonstrated that the gross electron signatures together with the observations of increasing (and also decreasing) $T_{e \perp}$ with increases (decreases) in magnetic field is explainable via the interaction of magnetized electrons in the intense, whistler-induced, short-scale, nonmonotonic magnetic fields occurring in the layer in a manner qualitatively similar to that reported by Hull et al. (2001). Namely, approximate separatrices, determined from magnetic moment and energy considerations, delineated quite well the electron pitch angle distribution topology according to source of electron orbits/contributions: those that can connect to both sides of the shock (free streaming orbits), the reflected orbits, as well as the trapped/excluded orbits due to local nonmonotonicities in the magnetic field. A detailed evaluation of how these features come about requires a more involved study beyond the scope of this current paper.

6. We showed evidence indicating that the anisotropies induced in the electron distributions via the lower-frequency whistlers are sufficiently strong to excite short spatial scale, quasi-parallel propagating, high-frequency whistlers near the electron resonance cone (near and above $100 \mathrm{~Hz}$ ). Note that we examined whether there is a connection with electron heat flux and found very little correspondence between peaks in electron heat flux and the occurrence of the HF whistlers in the interval.

The novel results shed light on the intricate coupling between the large-scale shock structure and small-scale interaction processes within the shock that can impact energization and thermalization processes. Whether this is characteristic of high beta, high-Mach number shocks, and how these features vary with shock conditions is not known and requires a thorough study of multiple events. 


\section{Appendix A: Determination of Shock Normal, Velocity, and Characteristic Parameters}

To determine the shock normal, speed, and characteristic parameters, we used the single-spacecraft least squares Rankine-Hugoniot method developed by Viñas and Scudder (1986), which is based on asymptotic upstream and downstream parameters. In this case we used fast survey ion density and velocities and survey magnetic field data from SC1. The survey magnetic field data were matched to ion moment time tags prior to performing the analysis. The upstream and downstream time intervals used are 0804:36 UT to 0805:12 UT and 0807:01 UT to 0808:21 UT, respectively. In this event 8 upstream and 18 downstream equally spaced points in these intervals from MMS1 were used to construct 144 permuted pairs of data for the optimization procedure to determine the normal and shock velocity. The shock normal was found to be $\hat{\mathbf{n}}_{\mathrm{RH}}=[0.9867,0.1615,-0.01916]$ and the shock velocity was found to be $25 \mathrm{~km} / \mathrm{s}$ along the normal. Once the shock normal and velocity are determined, the upstream and downstream asymptotic density, velocity, and magnetic fields were optimally determined for comparison with the measured average asymptotic data quantities. The shock parameters are given in Table A1. Shown are the asymptotic density, velocity components, and magnetic field components sampled in the upstream $\left(N_{1}, V x_{\mathrm{GSE} 1}, V y_{\mathrm{GSE} 1}, V z_{\mathrm{GSE} 1}, B x_{\mathrm{GSE} 1}, B y_{\mathrm{GSE} 1}\right.$, $\left.B z_{\mathrm{GSE} 1}\right)$ and downstream $\left(N_{2}, V x_{\mathrm{GSE} 2}, V y_{\mathrm{GSE} 2}, V z_{\mathrm{GSE} 2}, B x_{\mathrm{GSE} 2}, B y_{\mathrm{GSE} 2}, B z_{\mathrm{GSE} 2}\right)$ sides of the shock, respectively. Note vector components are in GSE coordinates in the spacecraft frame. The average and standard deviation of each of these quantities from moment and magnetic field data are given in the second and third columns, while the optimal fit results are given in the fourth column. The last column shows the ratio between the absolute deviation from the data average and optimal fit divided by the standard deviation. This ratio indicates that the difference between the fit and model agree to within 2 standard deviations. It is our experience that when the average upstream and downstream parameters from data are in good agreement with the optimal values determined from the R-H relations, the shock normal, and conservations equations are sufficiently well-determined.

To demonstrate that the shock coordinate system is reasonably well determined, Figure A1shows plasma and field data rotated in NIF coordinates. Note, the transformation to NIF is determined by the following unit vectors defined in GSE coordinates: the shock normal $\hat{\mathbf{n}}_{\mathrm{RH}}=[0.9867,0.1615,-0.01916]$, the out-of coplanarity component $\hat{\mathbf{s}} \equiv \hat{\mathbf{n}}_{\mathrm{RH}} \times \hat{\mathbf{b}}_{\mathbf{1}}=[-0.0267,0.2773,0.9604]$, and the third/tangential component $\hat{\mathbf{t}} \equiv \hat{\mathbf{n}}_{\mathrm{RH}} \times \hat{\mathbf{s}}=[0.1604,-0.9471,0.2779]$, with $\hat{\mathbf{b}}_{1}$ being the upstream magnetic field unit vector. The gray-shaded intervals indicate the upstream and downstream intervals used in the RH analysis. For context, the density and magnetic field amplitude are given in Figures A1a and A1b, respectively. The magnetic field vector components in NIF coordinates are shown in Figure A1c. The magnetic field data were smoothed using a $12 \mathrm{~s}$ boxcar sliding window to remove high-frequency structure from the shock macroscopic field (the

Table A1

Asymptotic Shock Parameters

\begin{tabular}{lcccc}
\hline Parameter & Average & $1 \sigma$ & Model fit & Ratio \\
\hline$N_{1}\left[\mathrm{~cm}^{-3}\right]$ & 6.573 & 0.202 & 6.540 & 0.1610 \\
$V x_{\mathrm{GSE} 1}[\mathrm{~km} / \mathrm{s}]$ & -708.2 & 2.704 & -709.2 & 0.3840 \\
$V y_{\mathrm{GSE} 1}[\mathrm{~km} / \mathrm{s}]$ & -7.515 & 8.522 & -5.546 & 0.2310 \\
$V z_{\mathrm{GSE} 1}[\mathrm{~km} / \mathrm{s}]$ & 17.91 & 5.713 & 17.48 & 0.07557 \\
$B x_{\mathrm{GSE} 1}[\mathrm{nT}]$ & -2.195 & 0.4931 & -2.510 & 0.6388 \\
$B y_{\mathrm{GSE} 1}[\mathrm{nT}]$ & 7.194 & 0.3717 & 7.767 & 1.542 \\
$B z_{\mathrm{GSE} 1}[\mathrm{nT}]$ & -1.482 & 1.327 & -2.313 & 0.6262 \\
$N_{2}\left[\mathrm{~cm}{ }^{-3}\right]$ & 21.86 & 1.245 & 22.06 & 0.1601 \\
$V x_{\mathrm{GSE} 2}[\mathrm{~km} / \mathrm{s}]$ & -207.4 & 11.59 & -205.8 & 0.1400 \\
$V y_{\mathrm{GSE} 2}[\mathrm{~km} / \mathrm{s}]$ & 82.37 & 13.76 & 79.13 & 0.2356 \\
$V z_{\mathrm{GSE} 2}[\mathrm{~km} / \mathrm{s}]$ & 5.772 & 17.02 & 7.041 & 0.07458 \\
$B x_{\mathrm{GSE} 2}[\mathrm{nT}]$ & -6.288 & 3.437 & -5.709 & 0.1683 \\
$B y_{\mathrm{GSE} 2}[\mathrm{nT}]$ & 28.63 & 3.547 & 26.66 & 0.5536 \\
$B z_{\mathrm{GSE} 2}[\mathrm{nT}]$ & 10.55 & 3.128 & -7.858 & 0.8618 \\
\hline
\end{tabular}




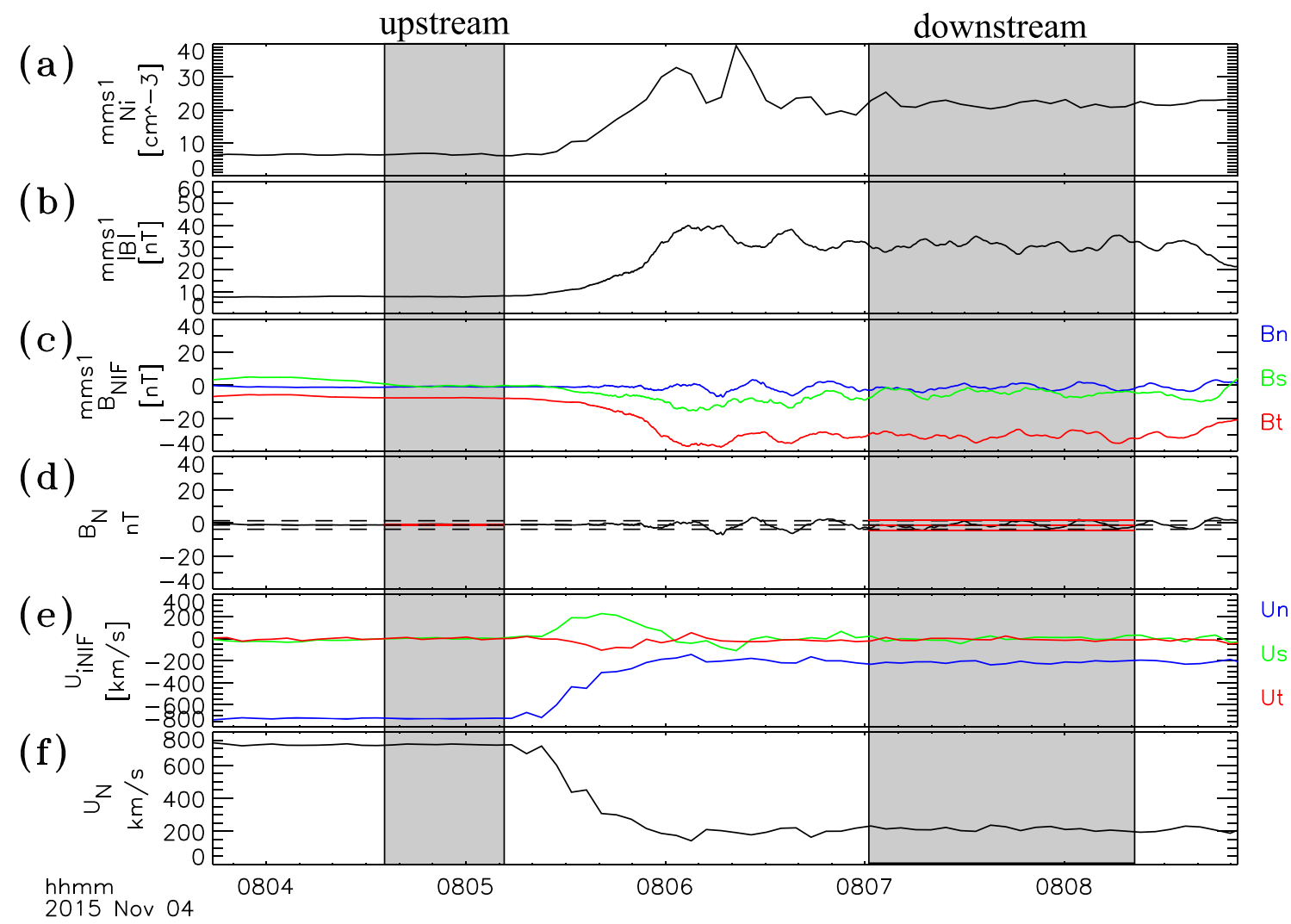

Figure A1. (a) Ion density, (b) magnetic field magnitude, (c) magnetic field components in NIF coordinates, (d) normal component of the magnetic field, (e) ion velocity in NIF, and (f) magnitude of the ion velocity along the normal. The two shaded regions indicate the upstream and downstream time intervals used for the RH analysis. The black dashed (and red) lines in (d) show the average and variance in the normal component over the respective intervals.

motivation behind the averaging window is discussed below). Consistent with a quasi-stationary description, the shock macroscopic magnetic field along the normal $B_{n}$ is reasonably constant with no sharp excursions anywhere in the interval. This is more clearly visible in Figure A1d, which depicts $B_{n}$ (solid curve) along with the RH optimal solution best fit and corresponding uncertainty (dashed lines). The solid red horizontal lines within each of the shaded intervals denote the respective average and variance of $B_{n}$. The largest variations in the normal magnetic field component are at and just past the overshoot region ( 0806:10 UT and after), which are much smaller than transverse component, with $\delta B_{n} / B_{t} \sim 0.09$ and thus are viewed to be reasonably small. The shock rest frame bulk velocity components in NIF coordinates and the magnitude are shown in Figures A1e and A1f, respectively. As expected, the upstream bulk velocity only has a component along the shock normal.

As a cross check, a four-spacecraft timing analysis method (Harvey, 1998) was used to determine the normal $\hat{\mathbf{n}}_{\mathrm{TOF}}$ and relative velocity $V_{\text {rel }}$ of the shock. This method, which depends on the relative spacecraft positions and signal time delays, is based on minimizing the function:

$$
S=\sum_{i=1}^{4} \sum_{j=1}^{4}\left[\hat{\mathbf{n}}_{\mathrm{TOF}} \cdot\left(\mathbf{r}_{i}-\mathbf{r}_{j}\right)-V_{\mathrm{rel}} t_{i j}\right]^{2},
$$

where $\mathbf{r}_{i}$ is the position vector of the $i$ th spacecraft and $t_{i j}=t_{i}-t_{j}$ is time delay of the signal between the $i$ th and $j$ th spacecraft. In order to apply this approach with the close separations of the MMS spacecraft, we used the magnitude of the magnetic field sampled at $16 \mathrm{~S} / \mathrm{s}$, which is of sufficient resolution to resolve the short time delays of the signal among the spacecraft. Time delays are determined via cross-correlation analysis applied to all possible pairings of the magnetic field magnitude in the interval (six independent in our case). To characterize the macroscale structure of the shock and reduce the effects of the nonstanding/nonstationary smaller-scale wave features present in the layer, the magnetic field data were boxcar smoothed prior to applying the technique. The normal and relative velocity were then computed as a function of averaging 
window. The time interval of data used was from 0805:18 UT to 0806:58 UT, which encompasses the full layer from the upstream to downstream side of the shock. Data from the other spacecraft were interpolated to MMS1's timeline prior to smoothing and performing the correlations. The optimal smoothing window was chosen where both the normal and relative velocity, which were computed as a function of averaging window, were reasonably stable. In this event a $12 \mathrm{~s}$ boxcar smoothing window was found to be a reasonable choice. The delay times are found to be $\delta t_{12}=-0.500 \mathrm{~s}, \delta t_{13}=-1.375 \mathrm{~s}, \delta t_{14}=-0.688 \mathrm{~s}, \delta t_{23}=-0.688$ $\mathrm{s}, \delta t_{24}=-0.125 \mathrm{~s}$, and $\delta t_{34}=0.4375 \mathrm{~s}$. The shock normal determined from multipoint timings was found to be $\hat{\mathbf{n}}_{\mathrm{TOF}}=[0.9905,0.1374,0.0005] \pm[0.0037,0.0208,0.0345]$ in GSE coordinates, which is in very good agreement with the $\mathrm{RH}$ result (angle between the TOF and $\mathrm{RH}$ normals is $2^{\circ}$ ). The shock velocity along the normal was found to be $v_{s h}^{\text {TOF }}=20 \pm 5 \mathrm{~km} / \mathrm{s}$, which is consistent with the RH result. Note, the errors in the normal shock and velocity were estimated by varying the averaging window $\pm 3 \mathrm{~s}$ and computing the standard deviation of timing results.

MVA was also applied to the smoothed magnetic field data. Shock normals from each spacecraft are determined to be $\hat{\mathbf{n}}_{\mathrm{mv} 1}=[0.9796,0.1356,-0.1486], \hat{\mathbf{n}}_{\mathrm{mv} 2}=[0.9838,0.1733,-0.0467], \hat{\mathbf{n}}_{\mathrm{mv} 3}=$ $[0.9804,0.1323,-0.1458]$, and $\hat{\mathbf{n}}_{\mathrm{mv} 4}=[0.9829,0.1342,-0.1259]$. The angles between the RH and MVA normals are found to be $\cos ^{-1}\left(\hat{\mathbf{n}}_{\mathrm{RH}} \cdot \hat{\mathbf{n}}_{\mathrm{mv} 1}\right)=7.6^{\circ}, \cos ^{-1}\left(\hat{\mathbf{n}}_{\mathrm{RH}} \cdot \hat{\mathbf{n}}_{\mathrm{mv} 2}\right)=1.7^{\circ}, \cos ^{-1}\left(\hat{\mathbf{n}}_{\mathrm{RH}} \cdot \hat{\mathbf{n}}_{\mathrm{mv} 3}\right)=7.5^{\circ}$, and $\cos ^{-1}\left(\hat{\mathbf{n}}_{\mathrm{RH}} \cdot \hat{\mathbf{n}}_{\mathrm{mv} 4}\right)=6.3^{\circ}$, which are in good agreement.

Other methods were also applied to cross-check the shock normal (e.g., Abraham-Shrauner, 1972). For example, using the single-spacecraft velocity coplanarity method, the shock normal can be estimated as $\hat{\mathbf{n}}_{\mathrm{VC}}= \pm\left(\mathbf{V}_{2}-\mathbf{V}_{1}\right) /\left|\mathbf{V}_{2}-\mathbf{V}_{1}\right|$, where $\mathbf{V}_{1}$ and $\mathbf{V}_{2}$ are upstream asymptotic velocities in any frame of reference. This method yields accurate normals for high Mach number shocks at nearly parallel or perpendicular geometries. Using this method, the normal was found to be $\hat{\mathbf{n}}_{\mathrm{VC}}=[0.9840,0.1766,-0.02385]$ in GSE coordinates, which agrees with the RH determined normal to within $1^{\circ}$. We also used the mixed data method, denoted as MD3 in Abraham Shrauner and Yun (1976), given by

$$
\hat{\mathbf{n}}_{\mathrm{MD} 3}= \pm \frac{\left[\left(\mathbf{B}_{2}-\mathbf{B}_{1}\right) \times\left(\mathbf{V}_{2}-\mathbf{V}_{1}\right)\right]}{\left|\left[\left(\mathbf{B}_{2}-\mathbf{B}_{1}\right) \times\left(\mathbf{V}_{2}-\mathbf{V}_{1}\right)\right]\right|},
$$

where $\mathbf{B}_{1}$ and $\mathbf{B}_{2}$ are upstream asymptotic magnetic field vectors measured in any frame of reference. Using this method, the normal is found to be $\hat{\mathbf{n}}_{\mathrm{MD} 3}=[0.9838,0.1776,-0.02427]$ in GSE coordinates, which also agrees with the RH determined normal to within $1^{\circ}$. Though these methods are a subset of the content in the optimal RH method, the close agreement indicates that these more simplified methods yield accurate normals in this event. These mutually consistent values estimated from the various methods and diagnostics presented in this section demonstrate that the shock normal is well determined and that the macroscale structure of the shock is quasistationary.

\section{Appendix B: Determination of Electron Separatrix Boundaries}

To identify the different classes of electron orbits affected by the nonmonotonic magnetic fields, we can use magnetic moment and energy constraints neglecting the effects of the cross-shock potential. In this approximation electrons at some location $x^{\prime}$ that pass through to some other point $x^{\prime \prime}$ must have parallel energies satisfying the condition $\mathcal{E}_{\|}(x)=\mathcal{E}_{\|}\left(x^{\prime}\right)+\mu\left(B\left(x^{\prime}\right)-B(x)\right)>0$ for all locations $x$ in the interval $\left[x^{\prime}, x^{\prime \prime}\right]$. This reduces to the sufficient condition that the absolute minimum parallel energy must satisfy: $\mathcal{E}_{\|}^{\min }=\mathcal{E}_{\|}\left(x^{\prime}\right)+\mu\left(B\left(x^{\prime}\right)-B^{\max }\right)>0$, where $B^{\max }$ is the maximum magnetic field value in the interval $\left[x^{\prime}, x^{\prime \prime}\right]$.

One can determine two types of separatrices by setting $\mathcal{E}_{\|}^{\min }=0$ : (1) the separatrix (denoted the mirror separatrix) that delineates electron orbits that can connect to both sides of the shock (free streaming orbits) and those that get reflected, and (2) the separatrix for trapped/excluded electron orbits. The mirror separatrix is obtained by requiring that the minimum electron parallel energy from a given location $x$ to the downstream side $x_{2}$ must vanish. The separatrix is defined as $\alpha_{\text {mirror }}=\sin ^{-1}\left[\sqrt{B(x) / B_{\circ}^{\max }}\right]$ for $\alpha \leq 90^{\circ}$ or $180^{\circ}-\sin ^{-1}\left[\sqrt{B(x) / B_{\circ}^{\max }}\right]$ for $\alpha>90^{\circ}$, where $B(x)$ is the magnetic field at the given location $x$, and $B_{\circ}^{\max }$ is the maximum shock macroscopic/background magnetic field within the interval $\left[x, x_{2}\right]$. Given that the interval depicted in Figure 7 is in the foot/ramp region, $B_{\circ}^{\max }$ corresponds to the overshoot in the shock macroscopic magnetic field estimated at $44 \mathrm{nT}$ (red curve in Figure 1a). The macroscopic field is used to account for the fact that superposed wave fields are likely transient features, which can significantly change during the spacecraft transit time through the shock. 
Separatrix boundaries delineating the trapping/exclusion regions were determined piecewise using nearest pairs of sufficiently large local magnetic field maxima bounding a given sampled location in the interval depicted in Figure 7. The boundaries are defined as $\alpha_{\text {trapped }}=\sin ^{-1}\left[\sqrt{B(x) / B_{*}^{\max }}\right]$ for $\alpha \leq 90^{\circ}$ or $180^{\circ}-$ $\sin ^{-1}\left[\sqrt{B(x) / B_{*}^{\max }}\right]$ for $\alpha>90^{\circ}$. To identify local trapping/exclusion regions, $B_{*}^{\max }$ is chosen to be the smaller of the two local magnetic maxima $B^{\max }\left(x^{\prime}\right)$ and $B^{\max }\left(x^{\prime \prime}\right)$ bounding a location $x$ within the given interval $\left[x^{\prime}, x^{\prime \prime}\right]$ of interest.

\section{Acknowledgments}

Funding for this work was provided by NASA grant 80NSSC17K0609. French involvement (SCM instruments) on MMS is supported by CNES and CNRS. We thank C. T. Russell for comments on the paper. We thank the MMS instrument teams and the MMS Science Data Center for providing the data. The MMS data used in this study are publicly available at the MMS Science Data Center website (https:// lasp.colorado.edu/mms/sdc/public/). Data from ACE are available at the NASA OMNIWeb website (https:/omniweb.gsfc.nasa.gov).

\section{References}

Abraham Shrauner, B., \& Yun, S. H. (1976). Interplanetary shocks seen by Ames plasma probe on Pioneer 6 and 7. Journal of Geophysical Research, 81(13), 2097-2102. https://doi.org/10.1029/JA081i013p02097

Abraham-Shrauner, B. (1972). Determination of magnetohydrodynamic shock normals. Journal of Geophysical Research, 77(4), 736-739. https://doi.org/10.1029/JA077i004p00736

Bale, S. D., Balikhin, M. A., Horbury, T. S., Krasnoselskikh, V. V., Kucharek, H., Möbius, E., et al. (2005). Quasi-perpendicular shock structure and processes. Space Science Reviews, 118, 161-203. https://doi.org/10.1007/s11214-005-3827-0

Balikhin, M. A., de Wit, T. D., Alleyne, H. S. C. K., Woolliscroft, L. J. C., Walker, S. N., Krasnosel'skikh, V., et al. (1997). Experimental determination of the dispersion of waves observed upstream of a quasi-perpendicular shock. Geophysical Research Letters, 24, 787-790. https://doi.org/10.1029/97GL00671

Balikhin, M. A., Walker, S. N., de Wit, T. D., Alleyne, H. S. C., Woolliscroft, L. J. C., Mier-Jedrzejowicz, W. A. C., \& Baumjohann, W. (1997). Non-stationarity and low frequency turbulence at a quasiperpendicular shock front. Advances in Space Research, 20(4/5), 729-734.

Burlaga, L. F., \& Ogilvie, K. W. (1973). Solar wind temperature and speed. Journal of Geophysical Research, 78, $2028-2034$.

Comisel, H., Scholer, M., Soucek, J., \& Matsukiyo, S. (2011). Non-stationarity of the quasi-perpendicular bow shock: Comparison between Cluster observations and simulations. Annales Geophysicae, 29, 263-274. https://doi.org/10.5194/angeo-29-263-2011

Dimmock, A. P., Balikhin, M. A., Walker, S. N., \& Pope, S. A. (2013). Dispersion of low frequency plasma waves upstream of the quasi-perpendicular terrestrial bow shock. Annales Geophysicae, 31, 1387-1395. https://doi.org/10.5194/angeo-31-1387-2013

Dimmock, A. P., Russell, C. T., Sagdeev, R. Z., Krasnoselskikh, V., Walker, S. N., Carr, C., et al. (2019). Direct evidence of nonstationary collisionless shocks in space plasmas. Science Advances, 5(2), eaau9926. https://doi.org/10.1126/sciadv.aau9926

Dunlop, M. W., Balogh, A., Glassmeier, K. H., \& Robert, P. (2002). Four-point Cluster application of magnetic field analysis tools: The curlometer. Journal of Geophysical Research, 107, 1384. https://doi.org/10.1029/2001JA005088

Dunlop, M. W., Southwood, D. J., Glassmeier, K. H., \& Neubauer, F. M. (1988). Analysis of multipoint magnetometer data. Advances in Space Research, 8, 273-277. https://doi.org/10.1016/0273-1177(88)90141-X

Ergun, R. E., Tucker, S., Westfall, J., Goodrich, K. A., Malaspina, D. M., Summers, D., \& Cully, C. M. (2016). The axial double probe and fields signal processing for the MMS mission. Space Science Reviews, 199(1), 167-188. https://doi.org/10.1007/s11214-014-0115-x

Eriksson, A. (1998). Spectral Analysis. In G. Paschmann, \& P. W. Daly (Eds.), Analysis methods for multi-spacecraft data pp. 5-42). Noordwijk, Netherlands: Eur. Space Agency.

Fairfield, D. H. (1974). Whistler waves observed upstream from collisionless shocks. Journal of Geophysical Research, 79, 1368-1378.

Fairfield, D. H., \& Behannon, K. W. (1976). Bow shock and magnetosheath waves at Mercury. Journal of Geophysical Research, 81(22), 3897-3906. https://doi.org/10.1029/JA081i022p03897

Feldman, W. C., Bame, S. J., Gary, S. P., Gosling, J. T., McComas, D. J., Thomsen, M. F., et al. (1983). Electron velocity distributions near the Earth's bow shock. Journal of Geophysical Research, 88(A1), 96-110.

Fitzenreiter, R. J., Scudder, J. D., \& Klimas, A. J. (1990). Three-dimensional analytical model for the spatial variation of the foreshock electron distribution function: Systematics and comparisons with ISEE observations. Journal of Geophysical Research, 95, 4155-4173.

Gary, S. P. (1993). Theory of space plasma microinstabilities, 7, Cambridge University Press, New York.

Harvey, C. C. (1998). Spatial gradients and the volumetric tensor. In G. Paschmann, \& P. W. Daly (Eds.), Analysis methods for multi-spacecraft data (pp. 307-322). Noordwijk, Netherlands: Eur. Space Agency.

Hellinger, P., \& Mangeney, A. (1997). Upstream whistlers generated by protons reflected from a quasi-perpdendicular shock. Journal of Geophysical Research, 102(A5), 9809.

Hull, A. J., Scudder, J. D., Fitzenreiter, R. J., Ogilvie, K. W., Newbury, J. A., \& Russell, C. T. (2000). Electron temperature and de Hoffmann-Teller potential change across the Earth's bow shock: New results from ISEE 1. Journal of Geophysical Research, 105(A9), 20,957-20,971.

Hull, A. J., Muschietti, L., Oka, M., Larson, D. E., Mozer, F. S., Chaston, C. C., et al. (2012). Multiscale whistler waves within Earth's perpendicular bow shock. Journal of Geophysical Research, 117, A12104. https://doi.org/10.1029/2012JA017870

Hull, A. J., Scudder, J. D., Larson, D. E., \& Lin, R. P. (2001). Electron heating and phase space signatures at supercritical, fast mode shocks. Journal of Geophysical Research, 106, 15,711-15,733.

Krasnosel'skikh, V., Balikhin, M. A., Alleyne, H. S. C., Klimov, S. I., Mierjedrzejowicz, W. A. C., Pardaens, A. K., et al. (1991). On the nature of low frequency turbulence in the foot of strong quasi-perpendicular shocks. Advances in Space Research, 11(9), 15-18.

Krasnoselskikh, V., Balikhin, M., Walker, S. N., Schwartz, S., Sundkvist, D., Lobzin, V., et al. (2013). The dynamic quasiperpendicular shock: Cluster discoveries. Space Science Reviews, 178, 535-598. https://doi.org/10.1007/s11214-013-9972-y

Krasnoselskikh, V. V., Lembége, B., Savoini, P., \& Lobzin, V. V. (2002). Nonstationarity of strong collisionless quasiperpendicular shocks: Theory and full particle numerical simulations. Physics of Plasmas, 9(4), 1192-1209. https://doi.org/10.1063/1.1457465

Le Contel, O., Leroy, P., Roux, A., Coillot, C., Alison, D., Bouabdellah, A., et al. (2016). The search-coil magnetometer for MMS. Space Science Reviews, 199(1), 257-282. https://doi.org/10.1007/s11214-014-0096-9

Lembege, B., Giacalone, J., Scholer, M., Hada, T., Hoshino, M., Krasnoselskikh, V., et al. (2004). Selected problems in collisionless-shock physics. Space Science Reviews, 110, 161-226. https://doi.org/10.1023/B:SPAC.0000023372.12232.b7

Lengyel-Frey, D., Hess, R. A., MacDowwall, R. J., Stone, R. G., Lin, N., Balogh, A., \& Forsyth, R. (1996). Ulysses observations of whistler waves at interplanetary shocks and in the solar wind. Journal of Geophysical Research, 101(A12), 27,555-27,564.

Lindqvist, P. A., Olsson, G., Torbert, R. B., King, B., Granoff, M., Rau, D., et al.(2016). The spin-plane double probe electric field instrument for MMS. Space Science Reviews, 199(1), 137-165. https://doi.org/10.1007/s11214-014-0116-9

Lobzin, V. V., Krasnoselkikh, V. V., Bosqued, J. M., Pincon, J. L., Schwartz, S. J., \& Dunlop, M. (2007). Nonstationarity and reformation of high-Mach-number quasiperpendicular shocks: Cluster observations. Geophysical Research Letters, 34, L05107. https://doi.org/10.1029/ 2006GL029095 
Matsukiyo, S., \& Scholer, M. (2003). Modified two-stream instability in the foot of high Mach number quasi-perpendicular shocks. Journal of Geophysical Research, 108(A12), 1459. https://doi.org/10.1029/2003JA010080

Matsukiyo, S., \& Scholer, M. (2006). On microinstabilities in the foot of high Mach number perpendicular shocks. Journal of Geophysical Research, 111, A06104. https://doi.org/10.1029/2005JA011409

Means, J. D. (1972). Use of the three-dimensional covariance matrix in analyzing the polarization properties of plane waves. Journal of Geophysical Research, 77, 5551-5559. https://doi.org/10.1029/JA077i028p05551

Muschietti, L., \& Lembège, B. (2017). Two-stream instabilities from the lower-hybrid frequency to the electron cyclotron frequency: application to the front of quasi-perpendicular shocks. Annales Geophysicae, 35, 1093-1112. https://doi.org/10.5194/angeo-35-1093-2017

Oka, M., Wilson III, L. B., Phan, T. D., Hull, A. J., Amano, T., Hoshino, M., et al. (2017). Electron scattering by high-frequency whistler waves at Earth's bow shock. Astrophysical Journal Letters, 842, L11. https://doi.org/10.3847/2041-8213/aa7759

Orlowski, D. S., \& Russell, C. T. (1991). ULF waves upstream of the Venus bow shock: Properties of one-hertz waves. Journal of Geophysical Research, 96(A7), 11,271-11,282. https://doi.org/10.1029/91JA01103

Pollock, C., Moore, T., Jacques, A., Burch, J., Gliese, U., Saito, Y., et al. (2016). Fast plasma investigation for magnetospheric multiscale. Space Science Reviews, 199(1), 331-406. https://doi.org/10.1007/s11214-016-0245-4

Robert, P., Dunlop, M. W., Roux, A., \& Chanteur, G. (1998). Accuracy of current density determination. In G. Paschmann, \& P. W. Daly (Eds.), Analysis methods for multi-spacecraft data (pp. 395-418) Noordwijk, Netherlands: Eur. Space Agency.

Rodriguez, P., \& Gurnett, D. A. (1975). Electrostatic and electromagnetic turbulence associated with the Earth's bow shock. Journal of Geophysical Research, 80, 19-31.

Russell, C. T., Anderson, B. J., Baumjohann, W., Bromund, K. R., Dearborn, D., Fischer, D., et al. (2016). The magnetospheric multiscale magnetometers. Space Science Reviews, 199(1), 189-256. https://doi.org/10.1007/s11214-014-0057-3

Scudder, J. D. (1995). A review of the physics of electron heating at collisionless shocks. Advances in Space Research, 15(8/9), 181-223.

Scudder, J. D., Mangeney, A., Lacombe, C., Harvey, C. C., Aggson, T. L., Anderson, R., et al. (1986). The resolved layer of a collisionless, high $\beta$, supercritical, quasi-perpendicular shock wave, 1. Rankine-Hugoniot geometry, currents, and stationarity. Journal of Geophysical Research, 91, 11,019-11,052.

Scudder, J. D., Mangeney, A., Lacombe, C., Harvey, C. C., Wu, C., \& Anderson, R. (1986). The resolved layer of a collisionless, high $\beta$, supercritical, quasi-perpendicular shock wave, 3. Vlasov electrodynamics. Journal of Geophysical Research, 91, 11,074-11,097.

Smith, C. W., Wong, H. K., \& Goldstein, M. L. (1991). Whistler waves associated with the Uranian bow shock: Outbound observations. Journal of Geophysical Research, 96(A9), 15,841-15,852. https://doi.org/10.1029/91JA01460

Stringer, T. E. (1963). Low-frequency waves in an unbounded plasma. Plasma Physics, 5, 89-107. https://doi.org/10.1088/0368-3281/5/2/ 304

Sundkvist, D., Krasnoselskikh, V., Bale, S. D., Schwartz, S. J., Soucek, J., \& Mozer, F. (2012). Dispersive nature of high mach number collisionless plasma shocks: Poynting flux of oblique whistler waves. Physical Review Letters, 108, 025002. https://doi.org/10.1103/ PhysRevLett.108.025002

Swanson, D. G. (1989). Plasma waves: Academic Press Inc.

Tidman, D. A., \& Krall, N. A. (1971). Shock waves in collisionless plasmas. New York: Wiley-Interscience.

Veltri, P., \& Zimbardo, G. (1993). Electron-whistler Interaction at the Earth's bow shock, 1. Whistler instability. Journal of Geophysical Research, 98, 13,325-13,333.

Viñas, A. F., \& Scudder, J. D. (1986). Fast and optimal solution to the Rankine-Hugoniot problem. Journal of Geophysical Research, 91 $39-58$

Walker, S. N., Balikhin, M. A., \& Nosdrachev, M. N. (1999). Ramp nonstationarity and the generation of whistler waves upstream of a strong quasiperpendicular shock. Geophysical Research Letters, 26(10), 1357-1360.

Wilson, L. B. III (2016). Low frequency waves at and upstream of collisionless shocks. In A. Keiling, D. H. Lee, \& V. Nakariakov (Eds.), Low-frequency waves in space plasmas (Vol.216, pp. 269-291). Washington, D. C: AGU. https://doi.org/10.1002/9781119055006.ch16

Wilson, L. B. III, Koval, A., Szabo, A., Breneman, A., Cattell, C. A., Goetz, K., et al. (2012). Observations of electromagnetic whistler precursors at supercritical interplanetary shocks. Geophysical Research Letters, 39, L08109. https://doi.org/10.1029/2012GL051581

Wilson, L. B. III, Koval, A., Szabo, A., Breneman, A., Cattell, C. A., Goetz, K., et al. (2013). Electromagnetic waves and electron anisotropies downstream of supercritical interplanetary shocks. Journal of Geophysical Research: Space Physics, 118, 5-16. https://doi.org/10.1029/ 2012JA018167

Wilson, L. B. III, Koval, A., Szabo, A., Stevens, M. L., Kasper, J. C., Cattell, C. A., \& Krasnoselskikh, V. V. (2017). Revisiting the structure of low-Mach number, low-beta, quasi-perpendicular shocks. Journal of Geophysical Research: Space Physic, 122, 9115-9133. https://doi. org/10.1002/2017JA024352

Wilson, L. B. III, Sibeck, D. G., Breneman, A. W., Contel, O. L., Cully, C., Turner, D. L., et al. (2014a). Quantified energy dissipation rates in the terrestrial bow shock: 1. Analysis techniques and methodology. Journal of Geophysical Research: Space Physics, 119, 6455-6474. https://doi.org/10.1002/2014JA019929

Wilson, L. B. III, Sibeck, D. G., Breneman, A. W., Contel, O. L., Cully, C., Turner, D. L., et al. (2014b). Quantified energy dissipation rates in the terrestrial bow shock: 2. Waves and dissipation. Journal of Geophysical Research: Space Physics, 119, 6475-6495. https://doi.org/ 10.1002/2014JA019930

Wu, C. S., Winske, D., Zhou, Y. M., Tsai, S. T., Rodriguez, P., Tanaka, M., et al. (1984). Microinstabilities associated with a high Mach number, perpendicular shock. Space Science Reviews, 37, 63-109.

Wu, C. S., Zhou, Y. M., Tsai, S. T., Guo, S. C., Winske, D., \& Papadopoulos, K. (1983). A kinetic cross-field streaming instability. Physics of Fluids, 26, 1259-1267.

Zhang, Y., Matsumoto, H., Kojima, H., \& Omura, Y. (1999). Extremely intense whistler mode waves near the bow shock: Geotail observations. Journal of Geophysical Research, 104, 449-461. 